In: European Journal of Neuroscience 44 (3): pp. 1935-1951. (2016) http://dx.doi.org/10.1111/ejn.13274 Manuscript as accepted This work is licensed under the Creative Commons Attribution-NonCommercialNoDerivatives 4.0 International License (CC BY-NC-ND 4.0).

\title{
Laminar analysis of the slow wave activity in the somatosensory cortex of anesthetized rats
}

Richárd Fiáth ${ }^{1,2,3}$, Bálint Péter Kerekes ${ }^{3}$, Lucia Wittner ${ }^{1}$, Kinga Tóth ${ }^{1}$, Patrícia Beregszászi ${ }^{3}$, Domonkos Horváth ${ }^{1,2,3}$, István Ulbert ${ }^{1,3}$

1) Institute of Cognitive Neuroscience and Psychology, Research Centre for Natural Sciences, Hungarian Academy of Sciences, Budapest, 1117, Magyar tudósok körútja 2.

2) School of Ph.D. Studies, Semmelweis University, Budapest, 1085, Üllői út 26.

3) Pázmány Péter Catholic University, Faculty of Information Technology and Bionics, Budapest, 1083, Práter utca 50/A

Corresponding author: István Ulbert

Institute of Cognitive Neuroscience and Psychology, Research Centre for Natural Sciences, Hungarian Academy of Sciences, Budapest, 1117, Magyar tudósok körútja 2., Hungary, tel: +36-1-382-6801 fax: +36-1-382-6295 email: ulbert.istvan@ttk.mta.hu

Running title: Laminar analysis of slow waves in the rat cortex

Keywords: local field potential, multiple-unit activity, current source density analysis, slow (< $1 \mathrm{~Hz}$ ) oscillation, slow wave activity 


\section{Abstract}

Rhythmic slow waves characterize brain electrical activity during natural deep sleep and under anesthesia, reflecting the synchronous membrane potential fluctuations of neurons in the thalamocortical network. Strong evidence indicates that the neocortex plays an important role in the generation of slow wave activity (SWA), however, contributions of individual cortical layers to the SWA generation are still unclear.

The anatomically correct laminar profiles of SWA were revealed under ketamine/xylazine anesthesia, with combined local field potential recordings, multiple-unit activity (MUA), current source density (CSD) and time-frequency analyses precisely coregistered with histology.

The up-state related negative field potential wave showed the largest amplitude in layer IV, the CSD was largest in layers I and III, while MUA was maximal in layer V, suggesting spatially dissociated firing and synaptic/transmembrane processes in the rat somatosensory cortex. Up-state related firing could start in virtually any layers (III-VI) of the cortex, but were most frequently initiated in layer V. However, in a subset of experiments, layer IV was considerably active in initiating up-state related MUA even in the absence of somatosensory stimulation. Somatosensory stimulation further strengthened up-state initiation in layer IV.

Our results confirm that cortical layer V firing may have a major contribution to the upstate generation of ketamine/xylazine-induced SWA, however, thalamic influence through the thalamorecipient layer IV can also play an initiating role, even in the absence of sensory stimulation. 


\section{Introduction}

Slow wave activity (SWA) emerging during sleep and anesthesia (Achermann \& Borbely, 1997; Steriade et al., 2001) is represented in cortical and thalamic neurons as a rhythmic alternation between depolarized (up-state) and hyperpolarized (down-state) states (Steriade et al., 1993a; Steriade et al., 1993c; Wilson \& Kawaguchi, 1996). Previous studies showed that SWA has a key role in restorative sleep functions, in the consolidation of memory traces and in the homeostatic regulation of synaptic strengths (Sirota et al., 2003; Born et al., 2006; Tononi \& Cirelli, 2006; Rasch \& Born, 2013; Tononi \& Cirelli, 2014). Slow waves are mostly of cortical origin, but the thalamus can also contribute to the generation of SWA under special circumstances (Steriade et al., 1993b; Crunelli \& Hughes, 2010; Luczak \& Bartho, 2012; David et al., 2013; Crunelli et al., 2015; Lemieux et al., 2014). In vivo laminar recordings of local field potentials (LFP) and multiple-unit activity (MUA) showed that SWA is generated in the infragranular layers of the cortex in animals (Toth et al., 2008; Sakata \& Harris, 2009; Chauvette et al., 2010). In vitro data and reports using optogenetic methods also support the predominance of infragranular layers in the generation of slow waves (Sanchez-Vives \& McCormick, 2000; Wester \& Contreras, 2012; Beltramo et al., 2013; Stroh et al., 2013). In contrast, human studies found that supragranular layers are more involved in the generation of SWA than infragranular layers (Cash et al., 2009; Csercsa et al., 2010).

The location of the neuronal activity gained from laminar multielectrode recordings was estimated from the physical depth of the electrode contacts below the cortical surface, and was related to corresponding anatomical data from the literature (Steriade \& Amzica, 1996; Sakata \& Harris, 2009; Chauvette et al., 2010; Kuki et al., 2015). No systematic histological analysis was performed on the same tissue, in order to precisely co-register the laminar electrical activity with the exact anatomical location of the given recording site(s). The combined analysis of 
current source density (CSD) and MUA was used to connect input and output features of local neuronal populations during evoked potentials (Mitzdorf, 1985) and sensory stimulation (Einevoll et al., 2007), but this important information is lacking during SWA.

In this study, we wished to elucidate the anatomically precise laminar origin of input and output attributes of SWA. Therefore, we investigated the laminar distribution of synaptic/transmembrane currents and neuronal firing activity during spontaneous and evoked up-states in the rat somatosensory cortex under ketamine/xylazine anesthesia, together with the anatomically verified positions of the electrode sites. 


\section{Materials and methods}

\section{$\underline{\text { Animal surgery and multielectrode implantation }}$}

Twenty Wistar rats (average: $308 \pm 88$ g, range: $180-460 \mathrm{~g}$, gender balanced) were used for the acute experiments. All experiments were performed according to the EC Council Directive of November 24, 1986 (86/89/EEC) and all procedures were reviewed and approved by the local ethical committee and the Hungarian Central Agricultural Office (license number: 22.1/4228/003/2009). Animals were anesthetized with a mixture of ketamine $(37.5 \mathrm{mg} / \mathrm{ml})$ and xylazine $(5 \mathrm{mg} / \mathrm{ml})$ intraperitoneally at $2 \mathrm{ml} / \mathrm{kg}$ body weight injection volume. Supplementary intramuscular ketamine/xylazine injections were given $(0.3 \mathrm{ml} / \mathrm{h})$ to maintain the depth of anesthesia during the recording sessions. Body temperature was retained at $37{ }^{\circ} \mathrm{C}$ with a homeothermic heating pad and a temperature controller (Supertech, Pécs, Hungary) throughout the experiments. After checking the absence of tail pinch and eyelid reflexes, rats were placed in a stereotaxic frame (David Kopf Instruments, Tujunga, CA, USA) and the skull was exposed by a small incision. The connective tissue between the skin and the skull was gently removed with a bone scraper. A $3 \times 3 \mathrm{~mm}$ window was drilled in the skull over the trunk region (S1Tr, $\mathrm{n}=15$, craniotomy: anterior-posterior (AP): $[-1.5-4.5]$, medial-lateral $(\mathrm{ML}):[+1+4]$ from the bregma) or over the hindlimb region (S1HL, $n=5$, craniotomy: AP: [0 -3], ML: $[+1.5+4.5]$ from the bregma) of the primary somatosensory cortex. The recording probe attached to a manual microdrive (David Kopf Instruments) was moved to the target location (S1Tr, AP: -2.7, ML: +3.3; S1HL, AP: -2, ML: +3 from the bregma according to Paxinos \& Watson (2007)) and inserted slowly ( $1 \mathrm{~mm} / \mathrm{min}$, driven by hand) to a depth of approximately $3 \mathrm{~mm}$ below the dura mater with guidance of a surgical microscope. The three most dorsal recording sites were positioned above the cortical surface using visual control. The probe was inserted at a $20^{\circ}$ angle from vertical in the coronal plane to position its shaft perpendicular to the brain surface and 
parallel to the dendritic tree of the cortical pyramidal cells. The dura mater was left intact during the experiment. Care was taken to avoid large blood vessels during probe implantation. Room temperature saline solution was dripped into the cavity of the craniotomy above the dura mater and was refilled every hour to prevent dehydration of the cortex.

\section{Electrophysiological recordings}

The details of the recording silicon multielectrode were described earlier in Grand et al. (2011). In short, the electrode had a $7 \mathrm{~mm}$ long shaft, with an $80 \times 280 \mu \mathrm{m}$ thickness, and had 24 square-shaped $(30 \times 30 \mu \mathrm{m})$ platinum contact sites with $100 \mu \mathrm{m}$ intercontact distances. Average site impedance and standard deviation of the recording sites at $1 \mathrm{kHz}$ were $343 \pm 66 \mathrm{k} \Omega$ (average of 9 probes), measured in Ringer's lactate solution (TEVA, Budapest, Hungary) against an $\mathrm{Ag} / \mathrm{AgCl}$ reference electrode with an electroanalytical activation and sensing instrument (EASI-1, BAK Electronics, Mount Airy, MD, USA).

We started recording one hour after the end of the surgery in order to let the cortex recover from the acute effects of the electrode insertion. Two stainless steel needles inserted into the left and right side neck muscles of the animal served as reference and ground electrodes. The brain signals were amplified and filtered (gain: 1000, band-pass: 0.1-7000 Hz) by a custommade headstage and amplifier (Ulbert et al., 2001), and were digitized at $20 \mathrm{kHz} / \mathrm{channel}$ sampling rate, on 24 channels at 16-bit precision (PCI-6259, National Instruments, Austin, TX, USA). The 24th channel was reserved for the registration of trigger signal containing the delivery time of the somatosensory stimuli. The recordings were carried out with customwritten LabVIEW software (National Instruments) and stored on the hard drive of a PC for offline analysis. 15-minute-long data segments were recorded and a recording session lasted about 3-5 hours. In four experiments, the recording was followed by administering a small positive current $(1.5 \mu \mathrm{A})$ for 1-2 minutes through three or four recording sites with well-defined 
distances from each other $(500,600,700$ or $800 \mu \mathrm{m})$ to form electric lesions and to accurately determine the relationship between recording sites and cortical laminae (Fig. 1A; Townsend et al., 2002).

\section{$\underline{\text { Somatosensory stimulation }}$}

Cutaneous receptive fields were identified by listening to the recorded signal in the 500$5000 \mathrm{~Hz}$ band through a pair of loudspeakers while using a cotton bud to tap the hindlimb and the trunk of the animal lightly, until the most intense responses of the area were found. Then this location was stimulated with a custom-made mechanical stimulator based on a stepper motor. The end of a thin wooden stick glued to the motor was placed $5 \mathrm{~mm}$ far from the skin of the rat for tapping stimulation. The parameters of the stimulator were controlled externally through a custom-made LabVIEW software. Duration of the pulses was either 50 or $100 \mathrm{~ms}$ long, which represents the period of time during which the stick reaches the skin of the animal, touches it and returns to the starting position. Angular velocities corresponding to the stimulus durations were $400^{\circ} / \mathrm{s}$ and $200 \%$, respectively. The stimulation frequency was set to $1.5 \mathrm{~Hz}$ based on the average peak frequency of ketamine/xylazine-induced SWA measured in our previous experiments. In one experiment, the interstimulus intervals were selected randomly from the $0.5-0.7 \mathrm{~s}$ range (corresponding to the $1.43-2 \mathrm{~Hz}$ frequency range). Stimulus delivery times were obtained from the trigger channel which recorded the stimulation pulses.

\section{$\underline{\text { Histology }}$}

After the recording session the multielectrode was withdrawn and the animal was deeply anesthetized and perfused through the heart first with physiological saline $(100 \mathrm{ml})$ followed by $4 \%$ paraformaldehyde in $0.1 \mathrm{M}$ phosphate buffer $(\mathrm{pH}=7.4,300 \mathrm{ml})$. The brain was removed and post-fixed overnight at $4{ }^{\circ} \mathrm{C}$. The fixed brain was sectioned using a vibratome (Leica 
VT1200, Leica Microsystems, GmbH, Wetzlar, Germany) into $60 \mu \mathrm{m}$ thick coronal sections. Following washing in $0.1 \mathrm{M}$ phosphate buffer, sections were mounted from gelatin, air dried, processed for cresyl violet (Nissl) staining, dehydrated in xylene (2x10 min) and coverslipped with DePex (SERVA Electrophoresis GmbH, Heidelberg, Germany). The sections were photographed under an Axioplan 2 microscope (Zeiss, Jena, Germany) equipped with a DP70 digital camera (Olympus, Tokyo, Japan) to examine the track of the silicon probe, to verify the recording position and to determine the boundaries between cortical layers (see Fig. 1). In case of several experiments $(n=4)$ the probes were dipped in red-fluorescent dye (DiI, $\sim 10 \%$ in ethanol, D-282, Life Technologies, Carlsbad, CA, USA) to accurately determine the position of the tip of the probe in the brain tissue (Fig. 1B). The gelatin-mounted and air dried sections were examined in the same Axioplan 2 microscope with AttoArc fluorescence illuminators using filters for Cy3 (exciting filter bandpass 530-560).

During histological processing the original size of the brain tissue may change. Depending on the histological procedure shrinkage or swelling of the sections can reach up to 10-25\% (Turner et al., 1995; Wellman et al., 1995; Pyapali et al., 1998; Tukker et al., 2013). A significant change in the size of the sections complicates the accurate determination of the relationship between cortical layers and recording sites, and may yield erroneous results. Therefore, to obtain the degree of tissue shrinkage or expansion in our case, we measured the change of the tissue size in the coronal plane along the mediolateral (x) and the dorsoventral (y) axes. To calculate the change in the x-axis, at the end of the experiment the retracted recording probe was reinserted laterally or medially into the brain tissue to well-defined distances $(600,700,800$ or $900 \mu \mathrm{m})$, parallel to the first insertion track ( $\mathrm{n}=4$ experiments; Fig. 1A). Afterwards, the distance between the two penetrations was measured on the Nissl-stained sections at two different positions which were separatsed at least $500 \mu \mathrm{m}$ apart from each other (Fig. 1A). The two distance values were averaged and compared with the known shift value. 
The size change in the y-axis was obtained by first measuring the spatial interval between the centers of adjacent electrical lesions visible on the Nissl-stained sections (Fig. 1A). The measured distance was then compared with the actual distance between those contact sites which were selected for inducing the lesions.

\section{$\underline{\text { Data analysis }}$}

The raw data were analyzed with the Neuroscan 4.3 software (Compumedics, El Paso, TX, USA) and using custom code written in MATLAB (MathWorks, Natick, MA, USA).

\section{Detection of cortical up- and down-state onsets}

Detection of up- and down-states from intracellular recordings is usually accomplished based on the bimodal distribution of the membrane potential (Volgushev et al., 2006), but state detection from extracellular signals is not a trivial task. Several methods were developed during the recent years, mostly based on the recorded local field potentials. Mukovski et al. (2007) used the gamma range $(20-100 \mathrm{~Hz})$ for detection, whereas Saleem et al. (2010) extracted the appropriate cortical states based on the phase of deep layer LFP recorded from the auditory cortex of anesthetized rats. In our earlier study we used the Hilbert-transformation to calculate the instantaneous phase of the narrow-band $(0.3-3 \mathrm{~Hz})$ filtered human LFP to extract up- and down-states (Csercsa et al., 2010). Sakata and Harris (2009) used the smoothed MUA for upstate detection.

Our method for the detection of the state onsets is shown in panel $\mathrm{C}$ of Figure 2. The algorithm was the following: we separated the MUA signal from the wideband data by filtering (band-pass filter, 500-5000 Hz, zero-phase shift, $24 \mathrm{~dB} /$ octave, rectification). Signals were downsampled from $20 \mathrm{kHz}$ to $2 \mathrm{kHz}$ for faster processing. The envelope of the downsampled MUA was extracted with an additional low-pass filter (zero-phase shift, $30 \mathrm{~Hz}, 24 \mathrm{~dB}$ /octave). 
After the filtering step, the values on the 23 channels were summed resulting in one trace representing the instantaneous intensity of summated population activity (SPA) in the examined cortical area. The amplitude histogram of the SPA showed a clear bimodal distribution (Fig. 2D). The threshold value to determine the up- and down-state onsets was calculated, as follows. The time points corresponding to the LFP peak of 50 randomly selected down-states (lack of MUA) longer than 100 ms were marked manually on the SPA trace and the average (AVG) and standard deviation (SD) of the selected values were calculated. The threshold level was set to $\mathrm{AVG}+(3 * \mathrm{SD})($ Fig. $2 \mathrm{C}$ and D). With lower threshold levels the algorithm may detect spurious up-state onsets within down-states, while higher threshold values can cause an inaccurate detection of state onsets. Based on empirical observations, the minimum duration of an up-state was set to $50 \mathrm{~ms}$ in the state detection algorithm, while the duration of a down-state was chosen to be minimum $100 \mathrm{~ms}$. The onset of an up (down)-state was defined as the time point at which the value of the SPA trace exceeded (fell below) the calculated threshold level. This time point had to be preceded by an opposite state with a length equal to or longer than the defined minimal state length.

\section{Estimation of the LFP gradient and the CSD}

The LFP reflects the summed synaptic activity of neuronal populations located in close vicinity to the recording electrode (Mitzdorf, 1985). To locate the position of macroscopic synaptic/transmembrane currents and to eliminate volume-conduction effects contaminating the field potentials, the LFP gradient (GRD) and the CSD was estimated from the LFP signal. The LFP was obtained from the wideband data by filtering (band-pass filter, 0.3-500 Hz, zerophase shift, $24 \mathrm{~dB}$ /octave). The GRD was computed to compare our results with that of a human study performed earlier (Csercsa et al., 2010). At each time instant the LFP gradient was estimated by the voltage difference between consecutive recording sites: 


$$
G R D_{j}=\left(u_{j+1}-u_{j}\right)
$$

where $u_{j}$ is the LFP in $\mu \mathrm{V}$ at a given recording site $j$. CSD analysis reveals synaptic/transmembrane generators of the LFP in laminated neural structures (Freeman \& Nicholson, 1975; Nicholson \& Freeman, 1975; Mitzdorf, 1985). The following three-point formula was used for the approximation of the CSD:

$$
\operatorname{CSD}_{j}=-\left(u_{j-1}-2 u_{j}+u_{j+1}\right) / r h^{2},
$$

where $u_{j}$ is the LFP in $\mu \mathrm{V}$ at a given recording site $j, r$ is the resistivity of the tissue in $\Omega^{*} \mathrm{~cm}$, and $h$ is the distance between recording sites (100 $\mu \mathrm{m}$ in our case). We assumed homogeneous tissue resistivity and both $r$ and $h$ were substituted by the dimensionless number 1 in the calculations. High spatial frequency noise and boundary effects were reduced by Hammingwindow smoothing and interpolation (Rappelsberger et al., 1981; Ulbert et al., 2001). Both the GRD and the CSD signal was calculated from the entire LFP trace and was processed the same way as LFP and MUA signals described in the next section. Both signal types were expressed in microvolts.

\section{Calculation of up-state onset locked depth profiles}

First, sections of the MUA and LFP traces containing artifacts were rejected. Next, the continuous LFP and MUA recordings and the estimated GRD and CSD signals were segmented into 1300-ms-long epochs beginning $500 \mathrm{~ms}$ before and ending $800 \mathrm{~ms}$ after the detected state onsets (set as time zero) for further up-state related analysis. A final manual artifact rejection and supervision process was performed on the epochs to exclude inadequate data. The remaining epochs were averaged together and depth profiles were created. Three groups were constructed and averaged based on the up-state duration histogram: brief (50-200 ms), average duration (200-400 ms) and long (> $400 \mathrm{~ms}$ ) up-states. For presentation purposes spatial 
interpolation was used between neighboring channels of up-state onset locked averages (cubic spline interpolation), and color maps were constructed from the interpolated data.

Normalized grand averages were calculated from all experiments as follows. The samples in the examined phases of up-states (up-state initiation, up-state peak, see Results for details) were averaged together within every channel. Next, the obtained values were averaged between the channels corresponding to the same cortical layer. Finally, these values were normalized and averaged between experiments. In case of the MUA, the background noise was calculated on every channel before normalization by averaging the MUA at the LFP peak of 50 randomly selected down-states (no unit activity). These noise values were then subtracted from the obtained MUA values.

\section{Detection of up-state onsets in different layers}

We aimed to locate the neocortical layer where cell firing starts during up-states. To achieve this we detected up-state onsets (see above) on every channel separately, except those with low MUA levels. Channels showing low firing activity were located in layers I, II and the bottom part of layer VI. These channels were disregarded from this analysis to avoid inaccurate results. Individual threshold levels were calculated for every channel, which were the sum of the average of down-state amplitudes on the actual channel $\left(\mathrm{AVG}_{\mathrm{j}}\right.$, where $j$ is the channel number and $j=1, . ., 23)$ and a constant value (C) obtained empirically. $\mathrm{AVG}_{\mathrm{j}}+\mathrm{C}$ resulted in similar threshold levels between the channels. The channel showing the largest fluctuations in the MUA level between up- and down-states (usually a channel located in cortical layer V with strong unit activity) was chosen as the reference channel. The time point corresponding to every up-state onset detected on the reference channel was then compared to the up-state onsets detected on the other investigated channels in a $300 \mathrm{~ms}$ time window and the channel with the 
earliest up-state onset was selected. Channels were then assigned to the appropriate cortical layers and the probability of up-state initiation indexed by MUA was calculated in each layer.

\section{Analysis of evoked up-states}

Up-states and down-states can be evoked with sensory, electric, magnetic or optical stimuli (Hasenstaub et al., 2007; Massimini et al., 2007; Vyazovskiy et al., 2009; Kuki et al., 2013). In this study, we used mechanical somatosensory stimulation to evoke up-states in the somatosensory cortex of anesthetized rats. We separated stimulus-evoked up-states from spontaneous ones as follows. Evoked up-states should have started in the interval of 10-60 ms after the onset time of the stimulus (stimulus delivered during the down-state) and had a minimum duration of $50 \mathrm{~ms}$ (the minimum duration of up-states set in our state detection algorithm). The 10-60 ms interval was selected empirically, after inspecting peristimulus time histograms showing the relation of the delivered stimulus and up-state onsets (Fig. 7B). All remaining up-states were considered spontaneous up-states. Responses to sensory stimuli delivered during down-states were usually brief events with moderate or low unit activity and with a duration of less than $50 \mathrm{~ms}$. These short responses were not analyzed in this study. After grouping, evoked up-states and spontaneous up-states were analyzed separately. Finally, CSD and MUA depth profiles of evoked up-states and spontaneous up-states were constructed and compared.

\section{Time-frequency analysis}

The averaged, relative spectrograms (Fig. 5G and H) were computed from LFP epochs with wavelet analysis implemented in MATLAB (Delorme \& Makeig, 2004) in a similar way as described in Csercsa et al. (2010). 
Statistical analysis

Statistica 11 (StatSoft, Tulsa, OK, USA) software was used for statistical analysis. Values are expressed as mean $\pm \mathrm{SD}$, or in the case of grand averages as mean \pm standard error $(\mathrm{SE}) \pm \mathrm{SD}$. The Kolmogorov-Smirnov test with Lilliefors significance correction was used for testing normality. Unpaired two-sample t-test was applied for the comparison of two populations of data. A P-value of 0.05 or less was considered significant. 


\section{Results}

$\underline{\text { Short description of the analysis procedure of recorded electrophysiological signals }}$

First, we computed the local field potential gradient (GRD) and current source density (CSD) from the wideband continuous signal. Our purpose behind the calculation of the GRD was to facilitate a direct comparison between the results obtained in this study and the findings of our earlier study investigating human SWA (Csercsa et al., 2010), where the GRD was recorded directly to minimize electro-magnetic artifacts. The CSD - the second spatial derivative of the LFP - estimates the transmembrane currents of the local neuronal population (Nicholson \& Freeman, 1975; Mitzdorf, 1985). The CSD analysis helped us to determine the input characteristics of the neurons located in the different layers of the neocortex during SWA. Next, we aimed to examine the basic spectral properties of neocortical SWA. We computed the laminar fast Fourier transform (FFT) power spectrum profile and the pairwise linear coherence from the continuous LFP and GRD traces in the frequency range of the SWA. Spectral data was calculated to verify the properties of SWA induced by ketamine/xylazine and to investigate the laminar distribution of the slow frequencies.

Laminar depth profile analyses of up-states were performed on 1300-ms-long epochs (see Materials and methods). These segments were grouped according to the duration of the upstate and averaged within the groups. Based on the anatomical reconstruction of the probe track, the recording channels were assigned to cortical layers. Color maps were computed using spatial interpolation to create the up-state onset locked laminar depth profiles. Finally, normalized grand averages were calculated from the data of all animals at two relevant phases of the up-states. 
$\underline{\text { Laminarization of the trunk and hindlimb region of the rat somatosensory cortex }}$

Our thorough anatomical analysis (Fig. 1A and B) revealed that the brain tissue showed a swelling of $\sim 2$ to $3 \%$ following histological procedures, both along the dorsoventral $(2.42 \pm 5.3$ $\%)$ and the mediolateral $(2.76 \pm 4.68 \%)$ axis. This would not substantially affect the results of our laminar analysis, and therefore, we did not apply any correction factor.

Channels recording the activity of the cortex were assigned to appropriate cortical layers based on the examination of Nissl-stained coronal sections and on the identification of the thalamorecipient layer IV with mechanical somatosensory stimulation. Contacts recording from above the dura mater or from below layer VI in the white matter were discarded. The six layers of the cortex were identified in Nissl-stained cortical sections (Fig. 1C). In the somatosensory cortex, layer I was a bright and cell-sparse area below the meninges and was approximately 150 $\mu \mathrm{m}$ thick (trunk region of the primary somatosensory cortex (S1Tr): $137 \pm 23 \mu \mathrm{m}$; hindlimb region of the primary somatosensory cortex (S1HL): $160 \pm 23 \mu \mathrm{m})$. This was followed by the thin, cell-dense layer II (S1Tr: $88 \pm 20 \mu \mathrm{m}$; S1HL: $95 \pm 16 \mu \mathrm{m})$. Layer III had an average thickness of around $400 \mu \mathrm{m}(\mathrm{S} 1 \mathrm{Tr}: 375 \pm 56 \mu \mathrm{m}$; S1HL: $401 \pm 59 \mu \mathrm{m})$. This layer contains mainly pyramidal cells and is usually merged together with layer II due to the lack of a clearly visible border between them. The thalamorecipient layer IV comprising a high number of spiny stellate cells, was an about $150 \mu \mathrm{m}$ thick, darker band located below layer III (S1Tr: $146 \pm 29 \mu \mathrm{m}$; S1HL: $143 \pm 18 \mu \mathrm{m})$. This was followed by layer $\mathrm{V}$ containing the cell bodies of the largest pyramidal cells in the rat somatosensory cortex. Layer $\mathrm{V}$ can be divided into two sublayers, the thinner and cell-sparser layer V/A (S1Tr: $142 \pm 36 \mu \mathrm{m}$; S1HL: 153 $\pm 24 \mu \mathrm{m})$ containing predominantly slender-tufted pyramidal cells (Schubert et al., 2006) and the approximately $300 \mu \mathrm{m}$ thick, celldense layer V/B (S1Tr: $300 \pm 59 \mu \mathrm{m}$; S1HL: $347 \pm 264 \mu \mathrm{m})$, mostly with a mixture of slender and thick-tufted pyramidal cells (Chagnac-Amitai et al., 1990; Kasper et al., 1994). Lastly, layer VI, found most ventrally and directly above the white matter, was the thickest of all the layers, 
with an average thickness of more than half a millimeter (S1Tr: $589 \pm 91 \mu \mathrm{m}$; S1HL: $669 \pm 136$ $\mu \mathrm{m}$ ). We found a significant difference between the thickness of the two investigated areas (Fig. 1D), the S1Tr $(1777 \pm 160 \mu \mathrm{m})$ and the S1HL $(1969 \pm 161 \mu \mathrm{m}, \mathrm{P}<0.001)$, which was caused predominantly by the thickness difference of layer V/B (300 $\mu \mathrm{m}$ vs. $347 \mu \mathrm{m}$, respectively, P < 0.025) and layer VI (589 $\mu \mathrm{m}$ vs $669 \mu \mathrm{m}$, respectively, $\mathrm{P}<0.037)$ between the two regions (Fig. 1E). 

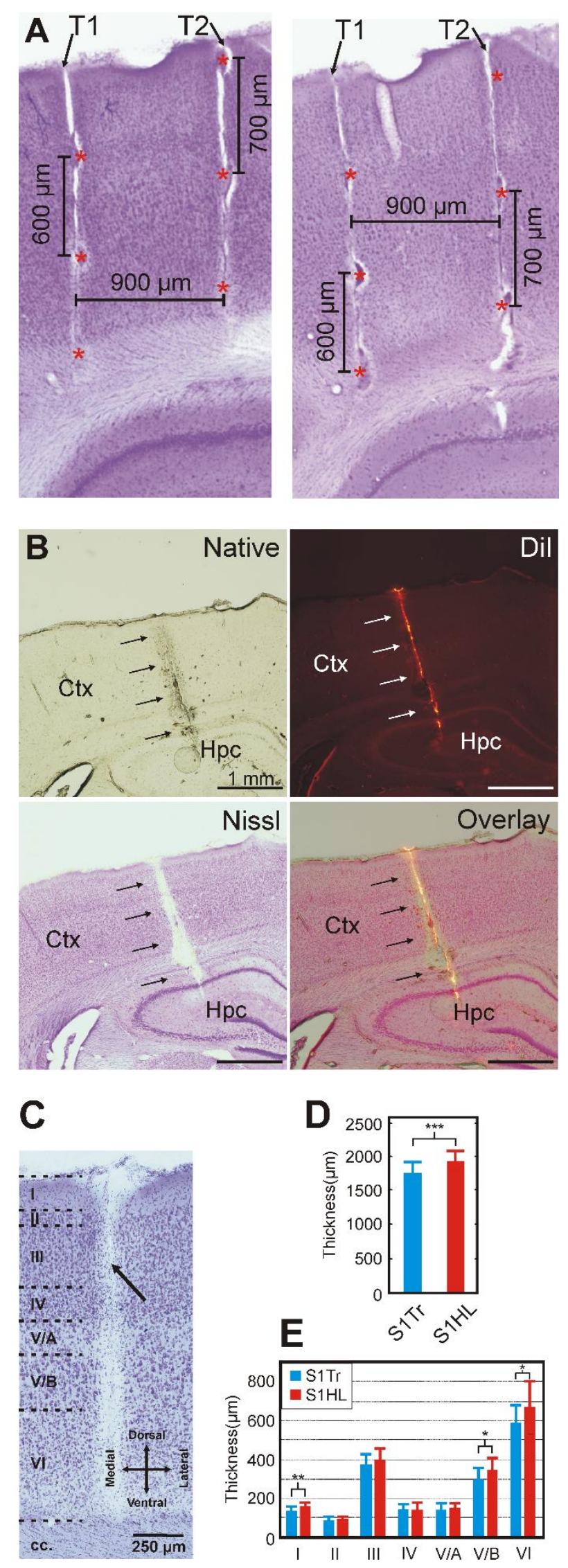

Figure 1. Methods used to determine the change in the size of the brain tissue during histological processing and the thickness of layers in the investigated cortical areas. (A) Two 
consecutive Nissl-stained coronal sections with two parallel electrode tracks (T1 and T2). The recording probe was inserted to a distance of $900 \mu \mathrm{m}$ from the first penetration for postmortem measurement of the mediolateral change in the size of brain tissue. The distances between small lesions (asterisks) created with electrical currents were used to determine the dorsoventral change in the tissue size. (B) Top left: Native image photographed under a light microscope before Nissl-staining. Top right: Fluorescent microscopy image of the same section with DiIstain showing the probe track. The photo was taken before Nissl-staining. Bottom left: The same coronal section after Nissl-staining containing the track of the silicon probe. Bottom right: the three images (native, fluorescent, Nissl) overlaid. Note that no visible change in the size of the tissue can be observed after Nissl-staining. The probe track is indicated with arrows on all images. (C) Nissl-stained coronal section from the trunk region of the primary somatosensory cortex (S1Tr) with the electrode track (black arrow). Boundaries between cortical layers are indicated with dashed black lines. Cortical layers are represented by roman numerals. cc. corpus callosum. (D) Average thickness of the SITr (measured in $n=23$ positions) and the hindlimb region of the primary somatosensory cortex (S1HL, $n=15$ positions). The trunk region was found significantly thinner compared to the hindlimb region (S1Tr vs. S1HL, $P=$ 0.0009, unpaired t-test). (E) The thickness of the six layers of S1Tr and S1HL. (S1Tr layer I vs. S1HL layer I, $P=0.0044 ;$ S1Tr layer II vs. S1HL layer II, $P=0.3072 ;$ S1Tr layer III vs. S1HL layer III, $P=0.1744$; S1Tr layer IV vs. S1HL layer $I V, P=0.7266$; S1Tr layer V/A vs. S1HL layer V/A, $P=0.2955$; S1Tr layer V/B vs. S1HL layer V/B, $P=0.0243$; S1Tr layer VI vs. S1HL layer VI, $P=0.0361$, unpaired t-tests). $* * * P<0.001, * * P<0.01, * P<0.05$. 
General temporal and spectral properties of the spontaneous slow wave activity in the $\underline{\text { somatosensory cortex of the anesthetized rat }}$

Detection of the onset of the two states alternating during SWA was performed with a method considering cell firing increase in the neocortex during up-states. The wideband recording (Fig. 2A) was filtered to obtain MUA (Fig. 2B), this latter providing the basis of the summated population activity (SPA, Fig. 2C). A clear bimodal distribution could be observed on the amplitude histogram of the SPA (Fig. 2D), where the two peaks of the histogram corresponded to the up-states and down-states, respectively. The threshold level calculated by our state detection algorithm (see Materials and methods for details and Fig. 2C and D for illustration) was located between the peaks corresponding to the two states. The onset time of every up- and down-state was determined to calculate the distribution of the duration of the states and to create state-locked averages.

A

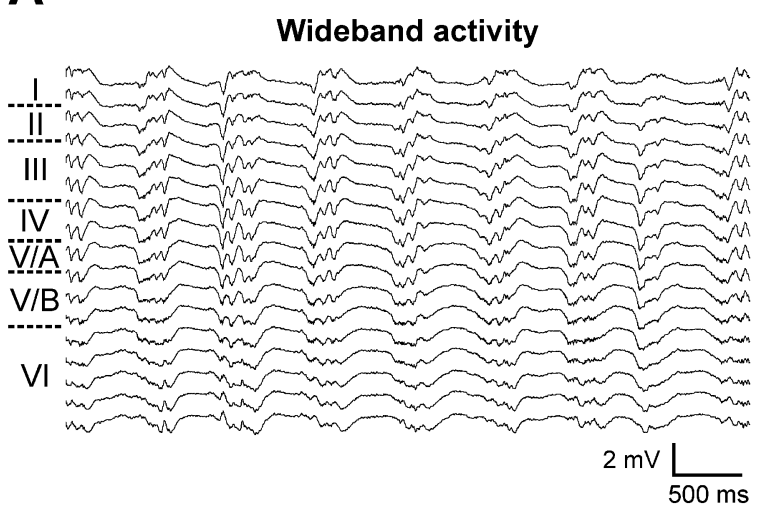

C Detection of up- and down-states

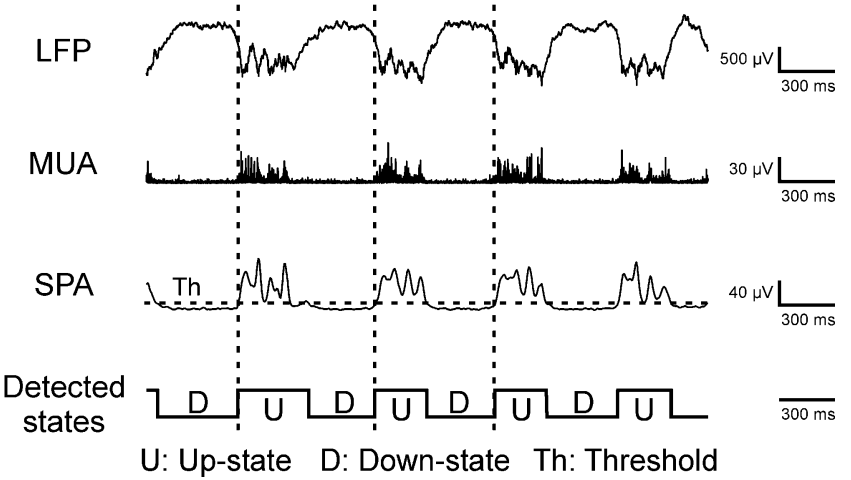

B

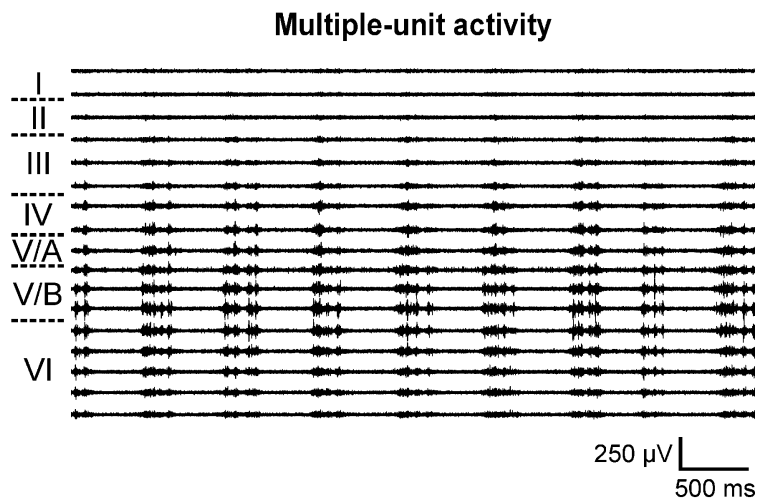

D Amplitude histogram of the SPA

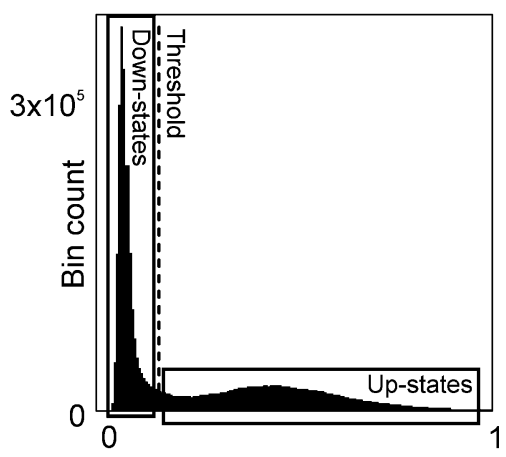


Figure 2. Representative five-second-long wideband activity (A) and multiple-unit activity $(M U A,(B))$ traces recorded with the silicon probe from the trunk region of the somatosensory cortex of the anesthetized rat. The ketamine/xylazine-induced slow wave activity is apparent both on the wideband signal (alternation of positive and negative potentials with $\sim 1.5 \mathrm{~Hz}$ ) and on the MUA (alternation of high and low unit activity with $\sim 1.5 \mathrm{~Hz}$ ) traces. Only channels which were located in the cortex are shown. The channels were assigned to cortical layers (roman numerals) based on the anatomical reconstruction of the probe track. (C) Detection of up- and down-state onset based on MUA traces. From top to bottom: first, the wideband signal was bandpass-filtered (500-5000 Hz, zero-phase shift, 24 dB/octave) and rectified to obtain the MUA. After that, the envelope of the unit activity was computed with an additional filtering step (30 Hz low-pass filter, zero-phase shift, $24 \mathrm{~dB} /$ octave) and cortical channels were summed up forming the summed population activity (SPA). Finally, a calculated threshold level was used to separate the up- and down-states (dashed horizontal line, 'Th'). Detected states are shown with horizontal bars below the traces. Vertical dashed lines mark some of the down-to-up transitions. (D) Amplitude histogram of the normalized SPA values showing bimodal distribution. The values of the narrow, high peak correspond to down-states (rectangle on the left), while values of the flat peak belong to up-states (rectangle on the right). The threshold level used for state detection is indicated with a dashed line.

Based on our data, up-states had an average duration of $292.77 \pm 111.73$ ms (range: 50 $894 \mathrm{~ms}$ ), while down-states were on average $305.29 \pm 94.17 \mathrm{~ms}$ long (range: $100-819 \mathrm{~ms}$ ). The average duration of a complete slow-wave cycle was $601.73 \pm 162.15 \mathrm{~ms}$ (range: $151-1473 \mathrm{~ms}$ ). Based on their duration, detected up-states were separated into three groups: brief (50-200 ms), average duration (200-400 ms) and long (> $400 \mathrm{~ms}$ ) up-states. These groups were analyzed independently. Most of the detected up-states $(n=35,520 / 56,263,61.8 \%)$ had an average 
duration, $20.4 \%(\mathrm{n}=11,629)$ were brief up-states, and the remaining $17.8 \%(\mathrm{n}=9,114)$ belonged to the group of long up-states. After artifact rejection and data cleansing (only upstates preceded and followed by down-states with a minimum length of $200 \mathrm{~ms}$ were further analyzed to avoid sections of the recordings containing faster oscillatory activity) the number of up-states described above decreased by an amount of about $30 \%$ (average duration up-states: $\mathrm{n}=24,513,66.6 \%$; brief up-states: $\mathrm{n}=5,434,14.8 \%$; long up-states: $\mathrm{n}=6,858,18.6 \%$ ).

The frequency of SWA under ketamine/xylazine anesthesia is somewhat higher compared to slow waves observed during natural slow-wave sleep and is usually more regular and rhythmic (Crunelli \& Hughes, 2010; Chauvette et al., 2011). We measured an average peak frequency of $1.5 \pm 0.26 \mathrm{~Hz}$ (range: $1.098-1.952 \mathrm{~Hz}$ ) during our experiments. This result was comparable to peak frequencies of the ketamine/xylazine-induced SWA found in rats by other groups (Sharma et al., 2010; David et al., 2013). The laminar FFT power spectrum profile in the frequency range of the SWA $(0.6-4 \mathrm{~Hz})$ calculated from the LFP and from the GRD of a representative experiment is shown in Figure $3 \mathrm{~A}$ and $\mathrm{B}$, respectively. The profile was constructed by spatial interpolation of the FFT power spectrum between cortical channels in the $0.6-4 \mathrm{~Hz}$ frequency band (cubic spline interpolation). The power of slow wave activity in the LFP was the strongest in layer I, IV and V, while it was lower in layer III and layer VI. The GRD power spectrum had two peaks, dominating in layer III and layer VI. This indicates that the LFP amplitude change between adjacent sites is maximal in these layers.

To examine the laminar distribution of the ketamine/xylazine-induced slow waves in more detail we calculated the pairwise linear coherence between each LFP channel and also between each GRD trace in the $0.6-3 \mathrm{~Hz}$ band (Fig. 3C and D). The depth distribution profile of the pairwise coherence of the LFP showed strong coherence between adjacent channels (Fig. 3C). Analyzing the depth profile of the pairwise coherence of the GRD channels demonstrated that there is a strong coupling within supragranular (I-III), granular (IV) and infragranular (V- 
VI) layers, but a considerable coherence was also confirmed between layer VI and layers II-III

(Fig. 3D). The weakest coherence was found between layer V and layer I.
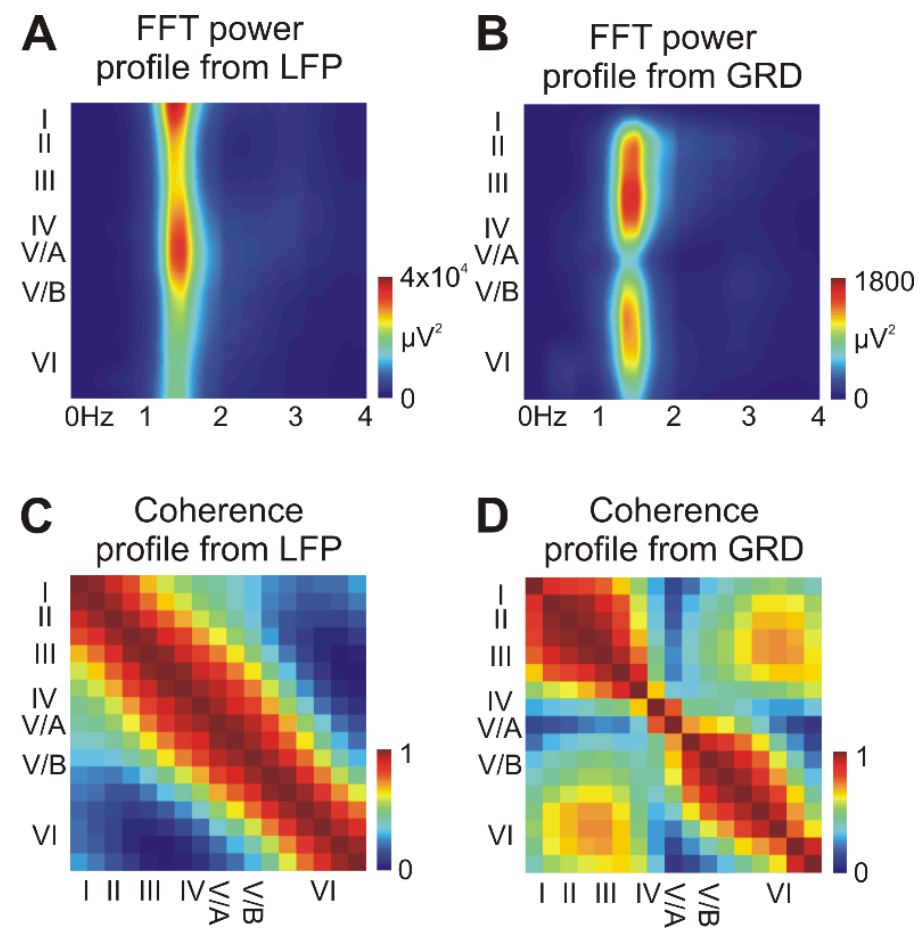

Figure 3. Temporal and spectral properties of the ketamine/xylazine-induced slow wave activity. (A) Representative depth profiles of the fast Fourier transform (FFT) power spectrum calculated from the local field potential (LFP). x-axis: frequency, y-axis: cortical depth, with corresponding layers, z-axis: color-coded FFT power. (B) Representative depth profiles of the FFT power spectrum calculated from the LFP gradient (GRD). x-axis: frequency, $y$-axis: cortical depth, with corresponding layers, z-axis: color-coded FFT power. (C-D) Representative depth distribution profile of pairwise coherence (range: 0.3-3 Hz) of the LFP (C) and GRD (D) channels located in different cortical layers. $x$-axis: cortical depth, with corresponding layers, y-axis: cortical depth, with corresponding layers, z-axis: color-coded pairwise coherence of the signals. 
Laminar depth profiles of spontaneous up-states recorded in the somatosensory cortex

After the detection of up-state onsets, 1300-ms-long epochs were cut from the continuous signals, with a 500-ms-long section before the up-state onset and an 800-ms-long section after the onset. Up-state onset locked averages were calculated from the LFP, GRD, CSD and the MUA traces and channels were assigned to the appropriate cortical layer (Fig. 4). Color maps were constructed from the average traces using spatial interpolation between neighboring channels (Fig. 5). Activity in the somatosensory cortex during up-states was frequently modulated by thalamocortical sleep spindles $(10-16 \mathrm{~Hz})$. Spindle cycles consisted of short ( 40ms), alternating phases of stronger and weaker MUA (see the traces in Fig. 2). Average duration up-states contained three or four of these spindle cycles (data not shown), but usually these were not visible on the averages, since they were not time locked to the up-state onsets.
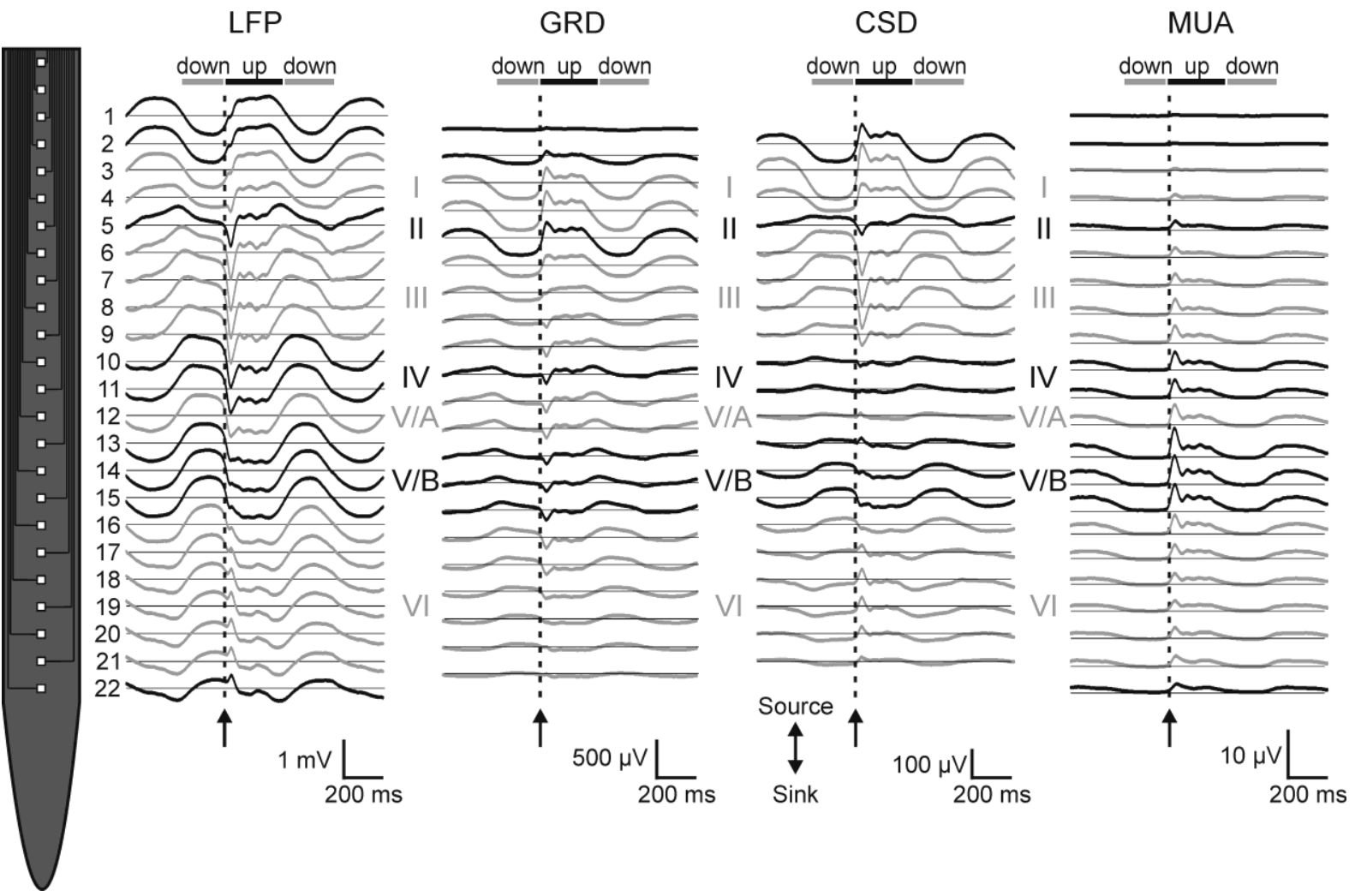
Figure 4. Depth profiles of the up-state onset locked averages of the recorded and derived electrophysiological signals from a representative experiment. Averages were calculated from up-states with average duration. Left - Schematic representation of the 24-contact silicon probe used for the experiments. The position of the probe is adjusted to the recordings next to it. Right - Up-state onset locked averages of the local field potential (LFP), LFP gradient (GRD), current source density (CSD) and multiple-unit activity (MUA). The average traces are 1300 ms long and the up-state onset is marked with an arrow and a dashed line. Horizontal bars above the traces indicate the extent of states (gray: down-state, black: up-state). The channels were assigned to cortical layers (roman numerals) based on the anatomical reconstruction of the probe track.
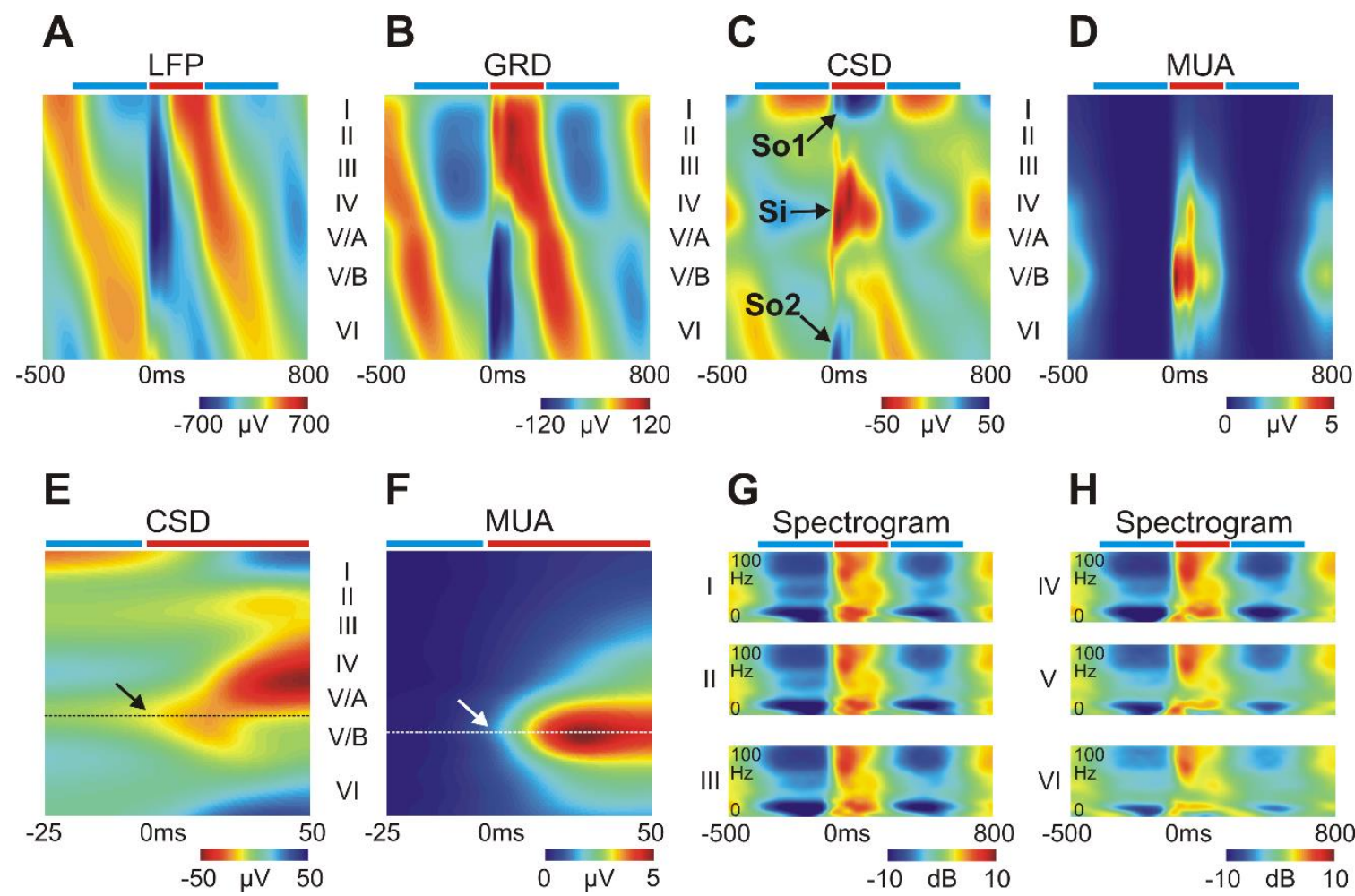

Figure 5. Up-state onset locked average depth profile maps constructed from up-states with average duration (200-400 ms) detected in a representative experiment. (A-D) Up-state onset locked depth profile maps after averaging the single sweeps ( $n=3082$ up-states). Time zero corresponds to the up-state onset. (A) local field potential (LFP), (B) LFP gradient (GRD), (C) 
current source density (CSD), (D) multiple-unit activity (MUA). x-axis: time, y-axis: cortical depth, with corresponding layers, z-axis: color-coded amplitude of the LFP, GRD, CSD and MUA units. Positive values are red, negative values are blue in case of the LFP and GRD. For CSD, sink is depicted in red and source in blue ('So1': supragranular source, 'Si': sink, 'So2': infragranular source). For MUA, low unit activity is colored blue, while high activity is depicted in red. CSD $(E)$ and MUA $(F)$ depth profile averages are shown at a faster timescale to demonstrate the laminar distribution of currents and unit-activity at the up-state onset. Note the appearance of the first sink and the initiation of the unit activity in layer $V$ during up-state onset (see arrows and dotted lines). (G-H) Up-state onset locked, averaged, relative spectrograms calculated from the LFP in the superficial $(G)$ and deep $(H)$ cortical layers. $x$ axis: time, y-axis: frequency, z-axis: color-coded averaged relative spectral power in $d B$. Horizontal bars above the depth profiles indicate the corresponding state (blue: down-state, red: up-state).

Normalized grand averages were calculated from all experiments at two relevant phases of the up-states (Fig. 6). The initial period of the up-state (termed as "up-state initiation", representing a 25 -ms-long time interval starting $25 \mathrm{~ms}$ after the up-state onset) was chosen because of the strongest currents and strongest MUA observed during this time interval (Fig. 6A). The other phase of the up-state, termed as "up-state peak", representing a 50-ms-long time interval starting $125 \mathrm{~ms}$ after the up-state onset, and corresponding to the positive LFP peak recorded in layer I during up-states (Fig. 6A) was also investigated. 
A

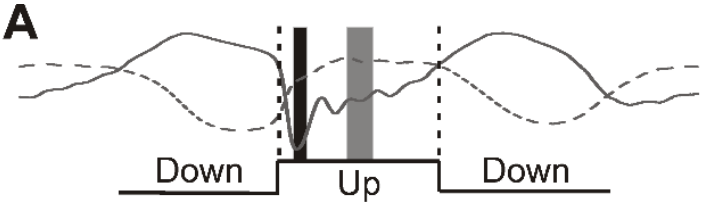

Up-state initiation $\quad$ Up-state peak ------- LFP (layer I)

(25-50 ms)

$(125-175 \mathrm{~ms}) \quad$ LFP (layer III)
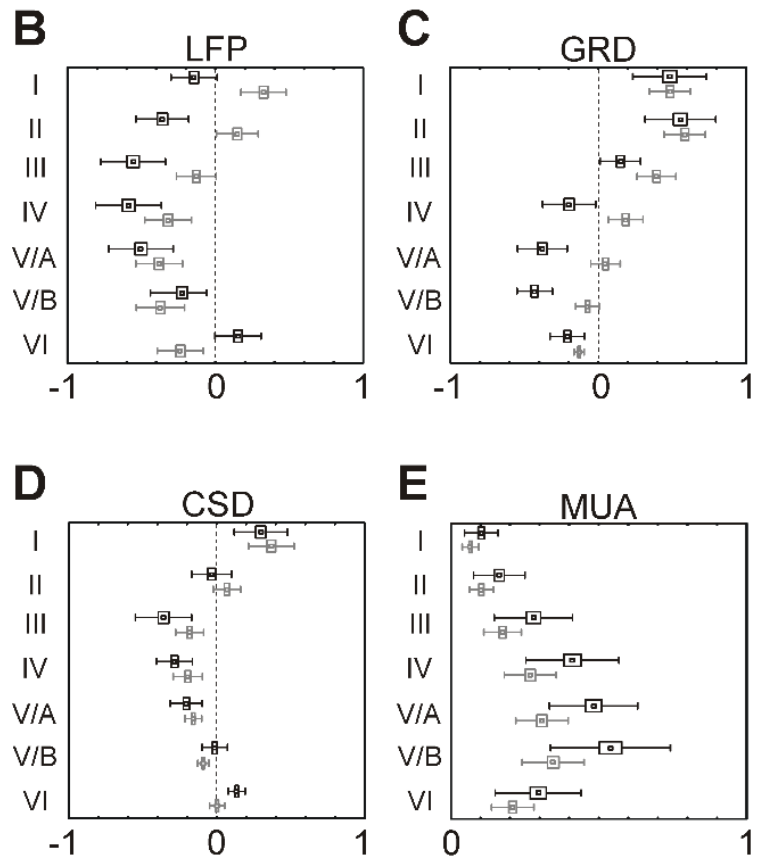

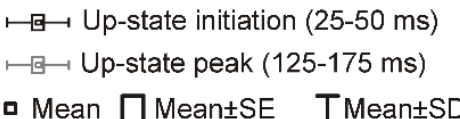

Figure 6. Up-state onset locked normalized grand averages of all experiments $(n=20) .(A)$

The two investigated phases of up-states. The initiation phase was a 25-ms-long interval starting shortly after the onset of the up-state, while the peak phase of the up-state was a 50ms-long interval set to the halftime of an up-state with average duration. (B-E) Box-whisker plots of the normalized grand averages of all rats at the initiation phase (25-50 ms, black) and at the peak phase of the up-state (125-175 ms, gray). (A) local field potential (LFP), (B) local field potential gradient (GRD), (C) current source density (CSD), (D) multiple-unit activity (MUA). Mean: small box, mean \pm standard error (SE): large box, mean \pm standard deviation (SD): whisker. 
A negative potential deflection was apparent in the depth profile of the LFP during the start of the up-state with an amplitude peak in layer IV and a polarity change in layer VI (Fig. 5A, Fig. 6B). After approximately $50 \mathrm{~ms}$, the field potential inverted into positive polarity in the superficial layers and remained positive until the subsequent down-state. The positivenegative polarity shift at the peak of the up-state was located at the top of layer III, close to the border of layer II and III. Below the supragranular layers the LFP amplitudes of up-states were negative. Analyzing the GRD revealed a positive deflection with a layer II peak shortly after the up-state onset which turned negative below the supragranular layers (Fig. 5B, Fig. 6C). The negative amplitude GRD had the strongest value in layer V. This deep negativity changed into positivity at the peak of the up-state in layer IV and in layer V/A as well, remaining negative only in layers V/B and VI. The depth profile of the CSD indicated a broad and strong current sink (inward current) during the beginning of the up-states, principally in layer III and IV, invading also the upper segment of layer V (Fig. 5C, Fig. 6D). We found that this presumably active sink (Fig. 5C, 'Si') was surrounded by two current sources (outward current), one stronger on the cortical surface (layer I, Fig. 5C, 'So1') and another weaker with a shorter duration in layer VI (Fig. 5C, 'So2'). This latter source vanished at the peak of the up-state, while the upper, layer I source persisted through the whole up-state. The strength of the sink decreased with the progression of the up-state and it shifted to deeper cortical layers. The MUA depth profile of up-state onset locked averages showed that the strongest MUA was formed in layer $\mathrm{V} / \mathrm{B}$ and the amplitude of the activity decreased both along the ventral and dorsal directions as a function of distance (Fig. 5D, Fig. 6E). In layer I and II we found only barely measurable MUA. At the beginning of the up-state, the MUA was approximately $30 \%$ stronger in every layer compared to the activity measured at the peak of the up-state (Fig. 6E). Both the depth profiles of the CSD and the MUA suggest that neuronal activity was the strongest during 
the first $50 \mathrm{~ms}$ of the up-state which corresponds to the first spindle cycle. After the first $50 \mathrm{~ms}$, the synaptic and spiking activity decreased with the progression of the up-state.

When we determined up-state onsets for every channel separately, we found that MUA started in layer V during the majority of up-states, in most cases ( $\mathrm{n}=16$ out of 20 experiments). The initiation of up-states was associated with a weak current sink located in layer $\mathrm{V}$ accompanied with early MUA in the same layer (Fig. 5E and F) on the up-state onset locked averages. Average spectrograms constructed from up-state onset locked epochs and computed in each layer showed a strong power increase in the $0.6-100 \mathrm{~Hz}$ frequency range during upstates, while a decrease in spectral activity could be observed during down-states, in agreement with previous data (Sakata \& Harris, 2009; Chauvette et al., 2010; Csercsa et al., 2010; Fig. 5G and $\mathrm{H})$.

The laminar depth profiles of brief and long up-states showed only minor differences compared to average duration up-states (data not shown). Analysis of LFP, GRD and CSD depth profiles of the down-states resulted in a similar pattern as that found during up-states, but with opposite polarities (data not shown). Laminar depth profiles of the MUA during downstates showed no detectable unit activity in any of the cortical layers, neither at the beginning, nor at the peak of the down-state. The main features of the depth profiles of down-states can be observed in the top row of Figure 5, where down-states precede and follow the investigated upstate.

\section{Laminar depth profiles of evoked up-states recorded in the somatosensory cortex}

In a subset of experiments $(\mathrm{n}=11$ out of 20 experiments $)$ rhythmic mechanical somatosensory stimulation was applied on the skin of the trunk or the hindlimb of the animal either with stimulation frequency similar to the peak frequency of the ketamine/xylazineinduced SWA $(1.5 \mathrm{~Hz})$ or with an interstimulus interval selected randomly from the $0.5-0.7 \mathrm{~s}$ 
range (see Materials and methods for details). Short sensory stimuli can evoke or terminate upstates depending on the instantaneous phase of the actual state (Hasenstaub et al., 2007). Our stimulation protocol could reliably evoke up-states (Fig. 7A and B). Stimulation of the receptive field of the investigated area resulted in evoked up-state ratios of 20-30\% of all detected upstates and occasionally the stimulation entrained the SWA for several seconds.
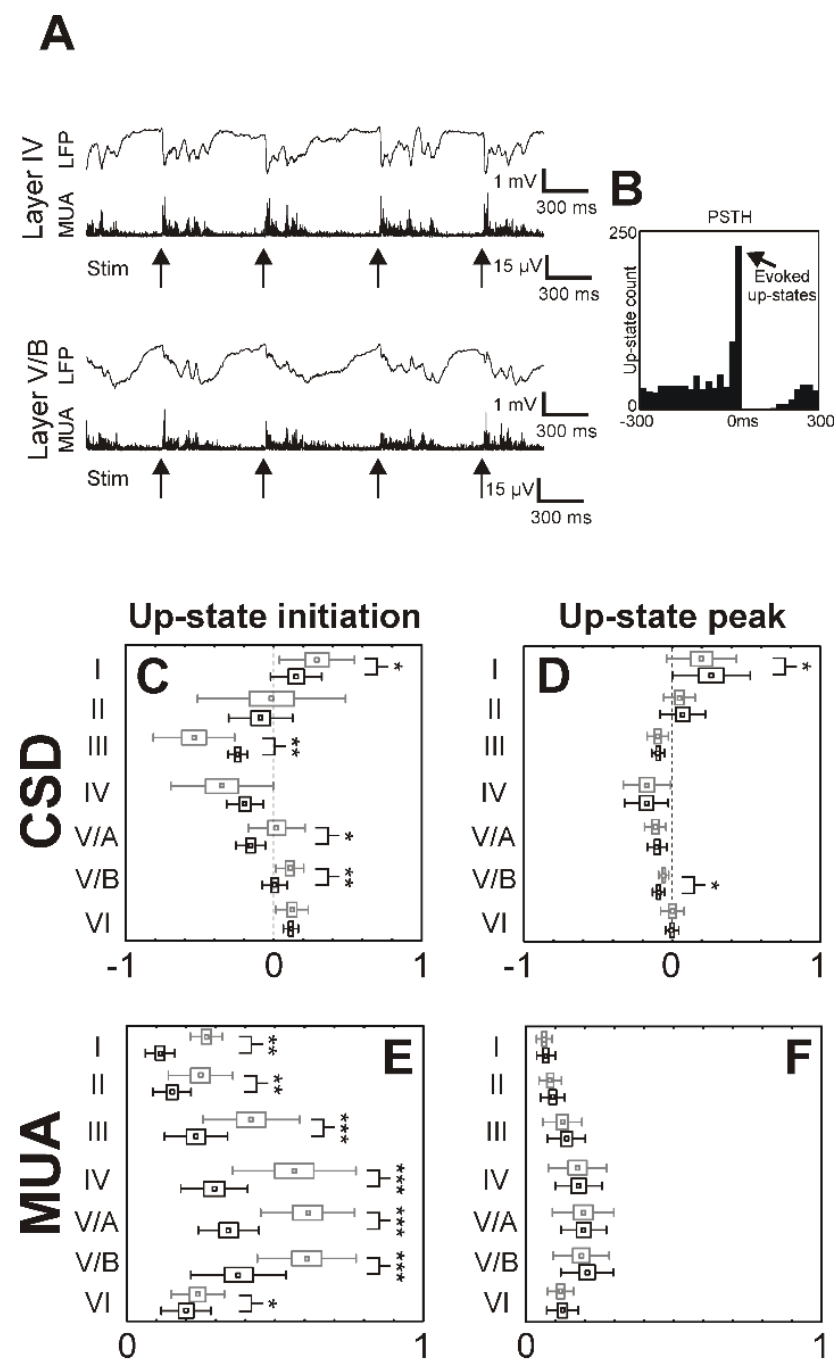

$$
\begin{aligned}
& \mapsto \text { Spontaneous up-state } \\
& \text {-⿴囗十 Evoked up-state } \\
& \text { - Mean } \square \text { Mean } \pm \text { SE IMean } \pm \text { SD }
\end{aligned}
$$

Figure 7. Laminar analysis of up-states evoked with mechanical somatosensory stimulation.

(A) Local field-potential (LFP) and multiple-unit activity (MUA) traces of representative consecutive evoked up-states recorded in layer IV and layer V/B of the somatosensory cortex. 
The time points of the stimulation are marked with black arrows. Stimulation was random in this representative example, with interstimulus intervals in the range between 500-700 ms. Note the steep slope of the LFP and the strong unit activity in the thalamorecipient layer IV during the onset of evoked up-states. (B) Peristimulus time histogram (PSTH) calculated from the time points of the up-state onsets and the delivered stimuli (bin size: $15 \mathrm{~ms}$ ). Note that a large amount of the up-states was initiated shortly ( 15-30 ms) after the stimulation (black arrow). These upstates were considered evoked up-states. (C-F) Up-state onset locked normalized grand averages of evoked (gray) and spontaneous (black) up-states $(n=11$ experiments) at the initiation phase (5-30 ms, $C$ and $E)$ and at the peak phase of up-states (125-175 ms, D and F). (C-D): current source density (CSD), (E-F): MUA. Mean: small box, mean \pm standard error (SE): large box, mean \pm standard deviation $(S D)$ : whisker. $* * * P<0.001, * * P<0.01, * P<$ 0.05

Evoked up-states were extracted from the data and their up-state onset locked CSD and MUA depth profiles were constructed (see Materials and methods for details). All the other upstates detected in recordings containing somatosensory stimulation were considered spontaneous up-states. Up-state onset locked CSD and MUA depth profiles of these spontaneous up-states were calculated as well and compared with the depth profiles of evoked up-states. The panels C-F in Figure 7 display the up-state onset locked normalized grand averages of evoked and spontaneous up-states, during the up-state initiation phase (5-30 ms) and at the peak of up-states (125-175 ms). We found that during the up-state initiation phase the recorded MUA during evoked up-states was significantly stronger in all layers compared to the unit-activity during spontaneous up-states (Fig. 7E). Significant differences in the CSD were also found in layers I, III and V, mainly related to stronger currents during the evoked up-states (Fig. 7C). This phenomenon is presumably caused by the synchronous activation of neuronal 
populations responsible for the generation of the transient evoked response to somatosensory stimulation. These differences in the synaptic and spiking activity vanished after the first $50 \mathrm{~ms}$ of up-states and the calculated CSD and MUA values of the two types of up-states became similar at the peak phase of the up-state (Fig. 7D and F). Another remarkable difference between the spontaneous and evoked up-states was that while in the former case a source-sink-source configuration characterized the initiation of up-states in the laminar profile of the CSD (Fig. 5C, Fig. 6D), in the latter case a new current source appeared in layer V (Fig. 7C). This current source may represent the passive recurrent flow related to the strong synaptic activity in layer IV and layer III induced by sensory stimulation. The earliest MUA onset was found in layer IV and the earliest sink was located in layer III and IV during stimulus evoked up-states, suggesting a strong thalamic contribution to these events through the thalamorecipient layer IV (Fig. 11).

\section{$\underline{\text { Laminar distribution of the multiple-unit activity onset during up-states }}$}

Our state detection method demonstrated that MUA during spontaneous up-states may start in any of the cortical layers (III-VI, Fig. 8). However, two cortical layers showed higher probability of up-state related firing onset than others. Usually, MUA began with the highest probability in layer V/B and IV (layer V up-states: Fig. 8A, Fig. 9; layer IV up-states: Fig. 8B, Fig. 10). MUA started with the highest probability (> 50\% of all detected up-states) in layer V/B (Fig. 8A, Fig. 9) during spontaneous activity in the majority of animals $(n=16 / 20$ experiments). Spontaneous up-states with layer IV MUA onset were found in high numbers, exceeding the number of layer $\mathrm{V}$ up-states (Fig. 8B, data from two experiments are shown) in several experiments $(n=4 / 20)$. 


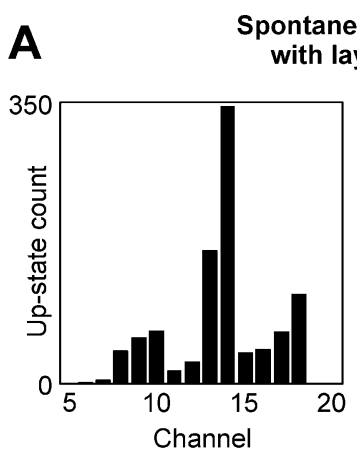

pontaneous up-states

with layer $\mathbf{V}$ onset

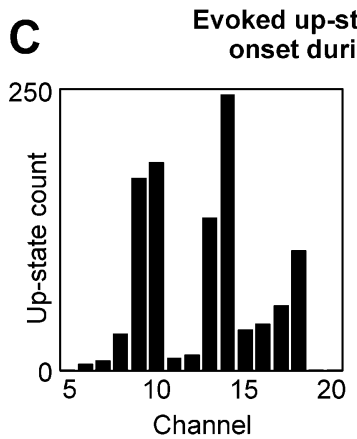

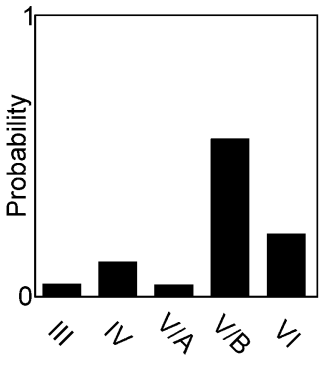

$$
\text { g stimulation }
$$

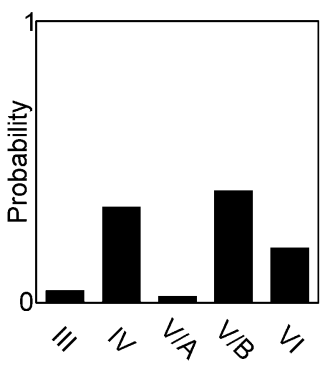

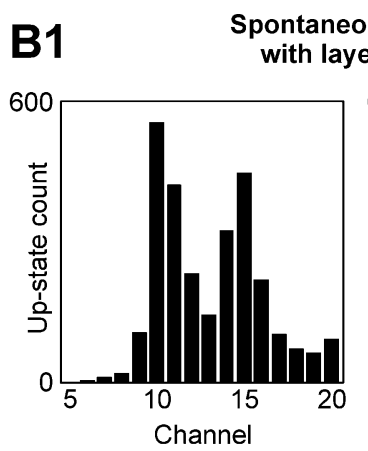
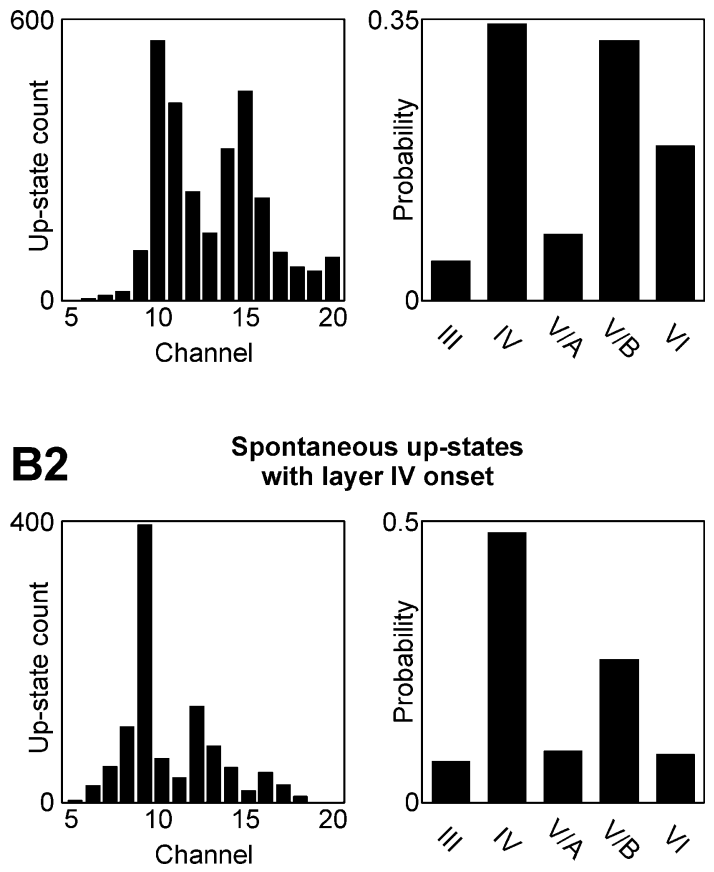

Figure 8. Multiple-unit activity (MUA)-based up-state onset in different cortical layers. (A)

Representative histogram showing the distribution of channels with the detected earliest MUA during spontaneous up-state onsets (left). Channels recording from layer I, II and from the bottom of layer VI were disregarded from the analysis because of low amplitude unit activity. Probabilities of spontaneous up-state initiation in different cortical layers (right) were derived from the histogram. Note that MUA during up-states started in layer V/B with a high probability, while probabilities for initiation in other layers were low. (B) The same as $(A)$, except these plots were derived from recordings containing spontaneous activity of two distinct experiments (B1 and B2). Note that the majority of MUA during spontaneous up-states started in layer IV instead of layer V/B. (C) The panel contains data from the experiment corresponding to panel (A). These plots were calculated from a recording containing somatosensory stimulation. Note that during stimulation the probability of MUA starting in layer V/B decreases with the simultaneous increase in the number of up-states starting in layer IV, presumably indicating evoked up-states. 
Furthermore, we examined the change in the laminar distribution of up-state onsets indexed by MUA in response to somatosensory stimulation. Compared to the results obtained during spontaneous activity, where mainly up-states with layer V onset dominated (Fig. 8A), the stimulation resulted in the increase of up-states starting in layer IV (Fig. 8C). This increase was accompanied by the simultaneous decrease of up-states with a MUA onset in layer V (cf. Fig. 8A vs. Fig. 8C). The majority of the detected up-states with a layer IV onset came shortly after a stimulus, indicating that they may belong to the group of evoked up-states (Fig. 11).
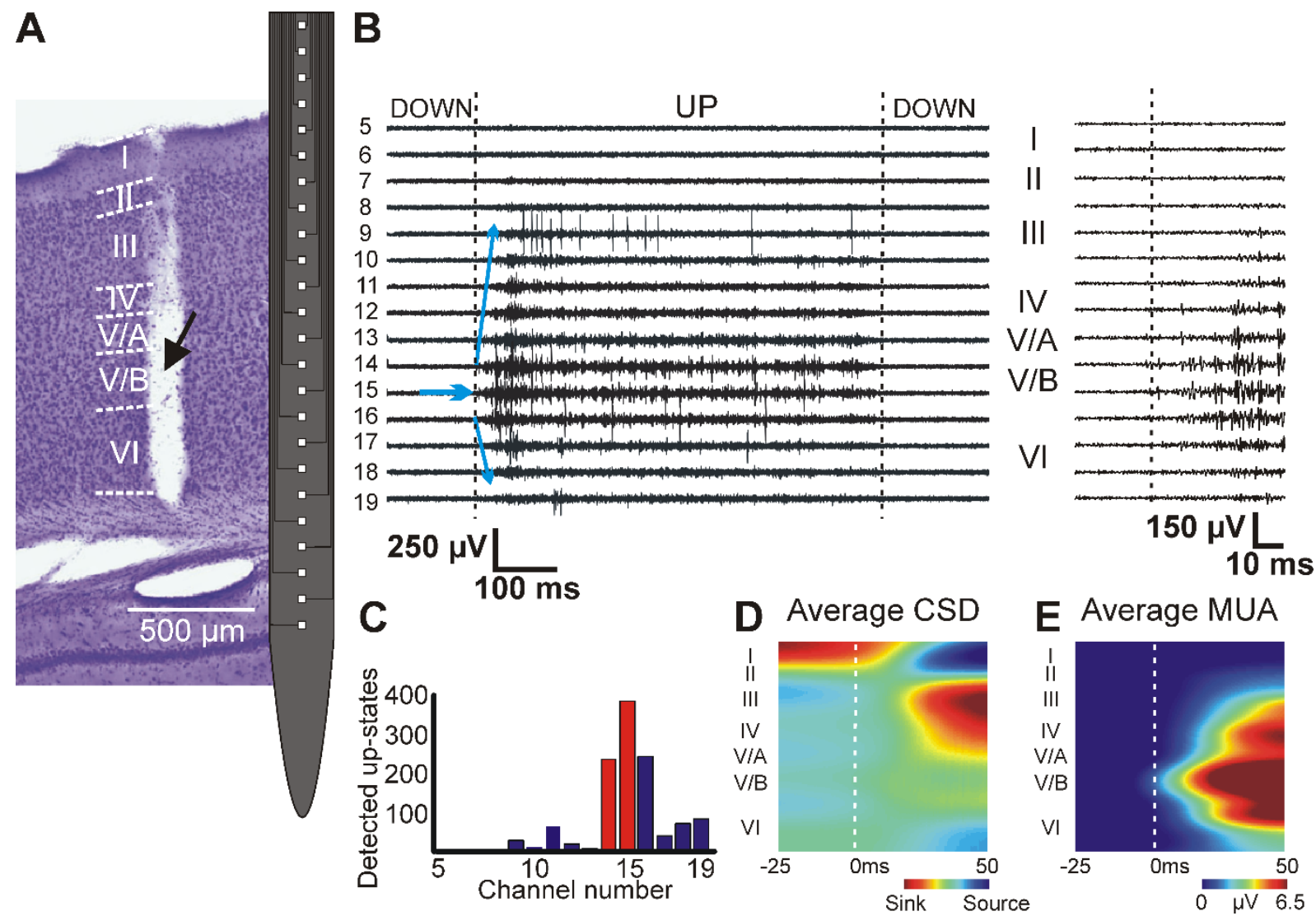

Figure 9. Spontaneous up-states with layer V onset. (A) Nissl-stained coronal section with the electrode track (black arrow) and the schematic of the silicon probe. Cortical layer boundaries are indicated with dashed white lines. Cortical layers are marked with roman numerals. (B) Left: one-second-long trace of cortical multiple-unit activity (MUA) recorded on 15 channels showing an up-state (UP) with layer V/B onset (starting on channel 15), which spreads toward 
superficial and deep cortical layers (indicated by blue arrows). Right: The onset of the up-state at a faster time-scale. (C) Up-state onsets detected on different channels of a 10-minute-long recording. From the 1230 up-states more than 600 started in layer V/B (channel 14, 15; red columns) based on the MUA. (D-E) Up-state onset locked current source density (CSD, D) and MUA (E) depth profile maps after averaging up-states with layer V/B onset. Time zero corresponds to the up-state onset. $x$-axis: time, $y$-axis: cortical depth, with corresponding layers, z-axis: color-coded amplitude of the CSD and MUA units. For CSD, sink is depicted in red and source in blue. For MUA, low unit activity is colored blue, while high activity is in red. Note the initiation of the unit activity in layer V/B during up-state onset.
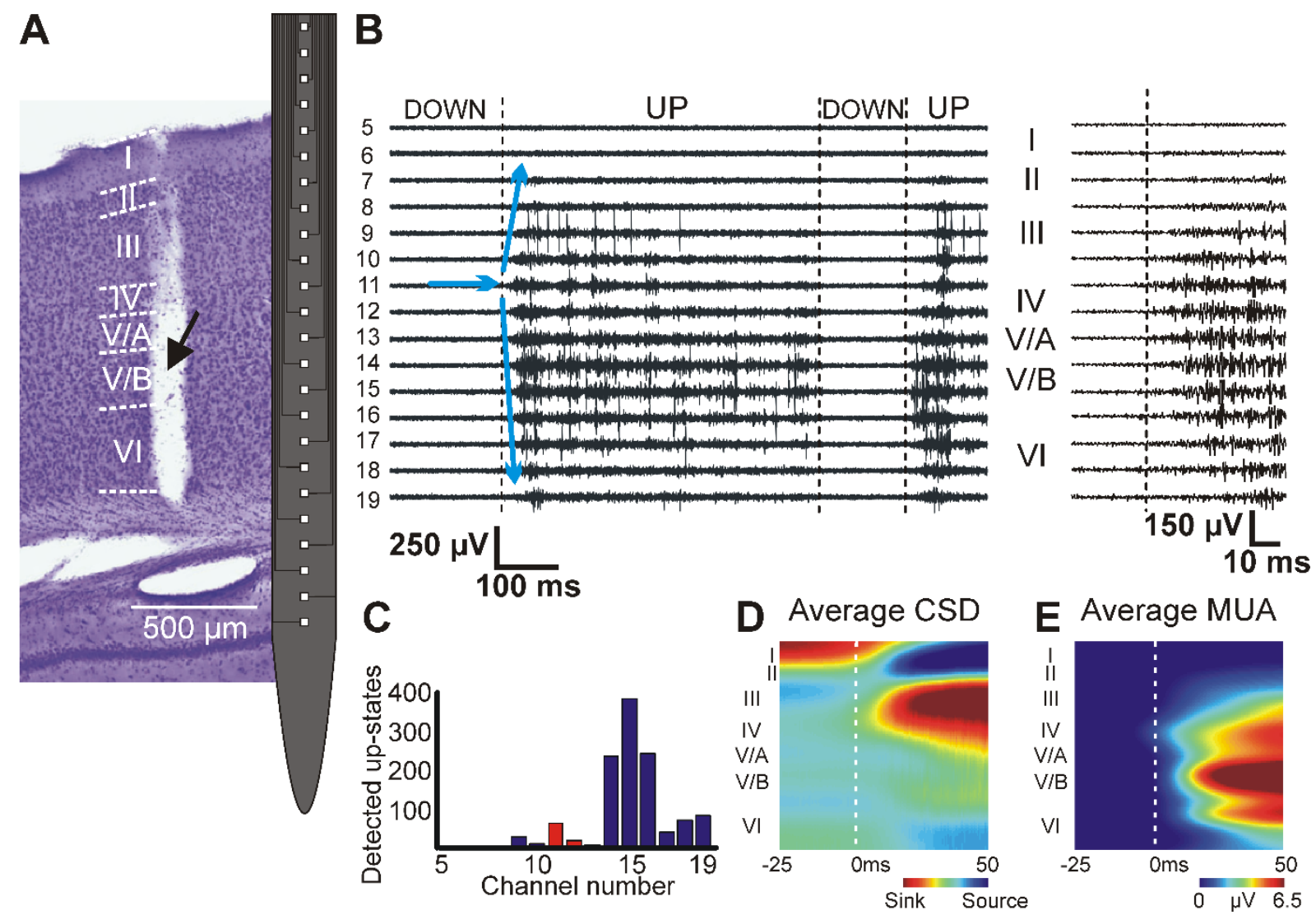

Figure 10. Spontaneous up-states with layer IV onset. (A) Nissl-stained coronal section with the electrode track (black arrow) and the schematic of the silicon probe. Cortical layer boundaries are indicated with dashed white lines. Cortical layers are marked with roman 
numerals. (B) Left: one-second-long trace of cortical multiple-unit activity (MUA) recorded on 15 channels showing an up-state (UP) with layer IV onset (channel 11), which spreads toward superficial and deep cortical layers (indicated by blue arrows). Note that the subsequent upstate was initiated in layer V. Right: The onset of the layer IV up-state at a faster time-scale. (C) Up-state onsets detected on different channels of a 10-minute-long recording (the same data that was used in Figure 9). From the 1230 up-states around 100 started in layer IV (channel 11, 12; red columns) based on the MUA. (D-E) Up-state onset locked current source density (CSD, D) and MUA (E) depth profile maps after averaging up-states with layer IV onset. Time zero corresponds to the up-state onset. x-axis: time, y-axis: cortical depth, with corresponding layers, z-axis: color-coded amplitude of the CSD and MUA units. For CSD, sink is depicted in red and source in blue. For MUA, low unit activity is colored blue, while high activity is in red. Note the initiation of the unit activity in layer IV during up-state onset and also earlier and stronger currents in the supragranular layers compared to Fig. 9D. 

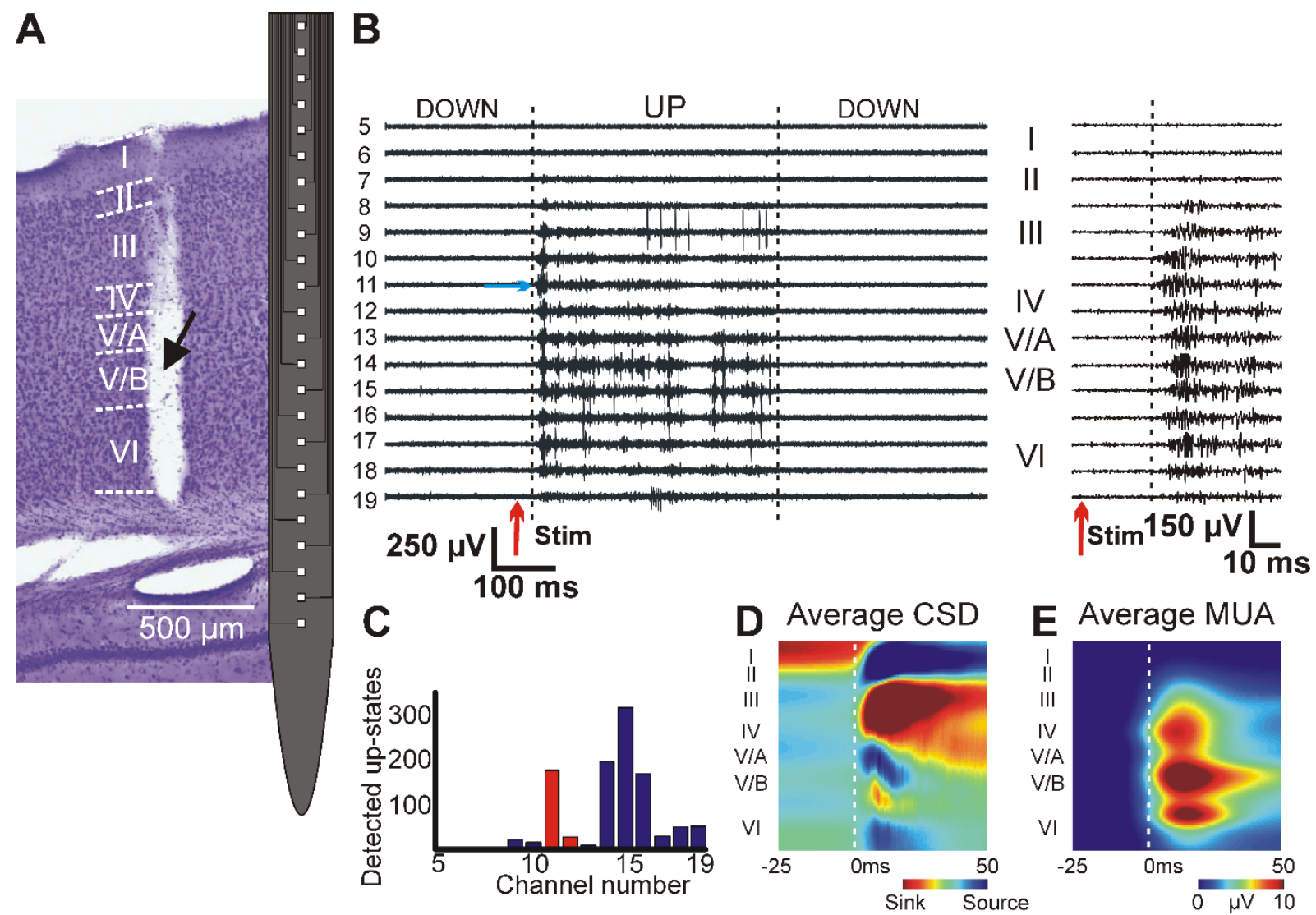

Figure 11. Up-states evoked by somatosensory stimulation. (A) Nissl-stained coronal section with the electrode track (black arrow) and the schematic of the silicon probe. Cortical layer boundaries are indicated with dashed white lines. Cortical layers are marked with roman numerals. (B) Left: one-second-long trace of cortical multiple-unit activity (MUA) recorded on 15 channels showing an evoked up-state (UP) with layer IV onset (channel 11) which appears rapidly in superficial and deep cortical layers. The time point of the stimulus onset is indicated with a red arrow. Right: The onset of the up-state at a faster time-scale. (C) Up-state onsets detected on different channels of a 10-minute-long recording. The skin of the animal was stimulated during the recording $(n=631$ stimuli). From the 1146 up-states around 191 were initiated in layer IV (channel 11, 12; red columns) based on the MUA. Furthermore, our algorithm detected 189 up-states evoked by stimulation. These results suggest that most of the up-states with layer IV onset were also evoked up-states. (D-E) Up-state onset locked current source density $(C S D, D)$ and MUA $(E)$ depth profile maps after averaging up-states with layer 
IV onset. Time zero corresponds to the up-state onset. $x$-axis: time, y-axis: cortical depth, with corresponding layers, z-axis: color-coded amplitude of the CSD and MUA units. For CSD, sink is depicted in red and source in blue. For MUA, low unit activity is colored blue, while high activity is in red. In this case most of the up-states with layer IV onset are actually evoked upstates, therefore the CSD distribution is different compared to spontaneous up-states which started in layer IV (Fig. 10D). The propagation of the MUA from the granular to supragranular and infragranular layers is faster (almost simultaneous) compared to spontaneous layer IV upstates (Fig. 10E vs. Fig. 11E). 


\section{Discussion}

In the present study, we investigated the laminar profile of SWA induced by ketamine/xylazine anesthesia in the primary somatosensory cortex of rats. Spontaneously occurring and evoked up-states were analyzed, combining wideband laminar multielectrode recordings and thorough histological examination. Analysis of the transmembrane currents revealed a strong and broad current sink in the middle layers during up-states, surrounded by two current sources with different durations. The longer source, located on the cortical surface, persisted through the entire up-state, while the shorter source lasted only for the first $50 \mathrm{~ms}$ of the up-state and was located in deep layers. We found that MUA was the most intense in layer $\mathrm{V}$ with a peak shortly after the initiation of the up-state. Down-states lacked spiking activity, except for some occasional firing of a few single units. Up-states elicited by mechanical somatosensory stimulation showed stronger MUA and transmembrane currents during the initiation phase compared to spontaneous up-states, but at the peak of the up-state the measured values became comparable. Our laminar recordings showed that neuronal populations started firing most frequently in layer $\mathrm{V}$ during the onset of up-states. However, we also found a substantial number of spontaneous up-states with layer IV onset, which might indicate a significant role of the thalamus in the generation of the SWA.

Precise co-registration of the electrophysiological data with the laminarization of the cortex is of high importance in order to accurately determine the spatial location of synaptic and unit activity during slow waves. Previous animal studies using linear multielectrodes related characteristics of slow waves to implantation depth (Steriade \& Amzica, 1996; Chauvette et al., 2010), or estimated the position of the electrode contacts in the neocortex with histological verification of the electrode track (Sakata \& Harris, 2009; Kuki et al., 2015). Both methods are prone to the possibility of inaccuracy, for several reasons. First, shrinkage or swelling of brain tissue may take place during histological processing, depending on the 
methods used (Pyapali et al., 1998; Gulyas et al., 1999; Tukker et al., 2013). Second, the tip of the recording electrode cannot be precisely positioned on the histological sections in several cases, resulting in the inaccurate estimation of the electrode position in the brain. In this study, we applied several methods to obtain the exact spatial relationship between the recording sites of the silicon probe and the cortical layers. The positive current administration at given electrode contacts and the reinsertion of the electrode at given distances helped us to determine tissue volume changes during histological procedures, whereas assignment of recording channels to the appropriate neocortical layers based on the track reconstruction provided a good solution to determine the accurate initiation site of transmembrane currents and cell firing during SWA.

\section{Laminar analysis of the slow waves in animal models}

Slow wave activity has been thoroughly investigated in several animal models both under anesthetic conditions and during natural sleep (Sanchez-Vives \& McCormick, 2000; Sakata \& Harris, 2009; Chauvette et al., 2010; Sanchez-Vives et al., 2010; Sharma et al., 2010; Chauvette et al., 2011; Luczak \& Bartho, 2012; David et al., 2013; Crunelli et al., 2015; Lemieux et al., 2014; Sheroziya \& Timofeev, 2014; Lemieux et al., 2015). The majority of studies so far showed the key role of the neocortex in the generation and maintenance of the SWA with the thalamus as an important supporting actor (Crunelli \& Hughes, 2010; David et al., 2013; Crunelli et al., 2015; Lemieux et al., 2014). However, the laminar origin of cortical generators of the slow rhythm was not well described and there are some contradictions in the existing literature.

Several properties of the ketamine/xylazine-induced slow waves recorded in our experiments were comparable to the findings of other groups, including the peak frequency of the slow oscillation (Sharma et al., 2010) and the duration of the states (Volgushev et al., 2006; 
Saleem et al., 2010). The first SWA-related CSD analysis studies were performed in cortical area 5 of ketamine/xylazine anesthetized cats (Steriade \& Amzica, 1996). Steriade and Amzica found a remarkable current sink in the middle layers between two sources located in the upper and deeper layers during the up-state of the SWA, which is in good agreement with our findings. However, a weaker sink was also present in the deepest layer of the neocortex, which was absent on the CSD depth profiles we obtained. In another study using laminar silicon multielectrodes implanted into neocortical areas 5, 7 and 21 of naturally sleeping cats, Chauvette and colleagues observed up-state-related current sinks in the deeper layers and sources in the upper layers of the cortex (Chauvette et al., 2010), a similar distribution compared to our results.

CSD analysis of spontaneous slow waves induced by the anesthetic urethane in the hindlimb area of the somatosensory cortex of rats revealed a strong sink in layer III and two weaker ones with longer latency in layer V and VI during up-states, while current sources were found in layer I/II and VI (Toth et al., 2008). Again, except for the deep, layer VI sink, these findings agree well with the CSD depth profile maps shown in our study. The missing sink located in the deep layers may be the consequence of different anesthetic agents used. Slow waves emerging during urethane anesthesia are longer in duration and are less regular compared to SWA induced by the administration of ketamine/xylazine (Crunelli \& Hughes, 2010). In another study, Sakata and Harris (2009) investigated the spontaneous MUA in the auditory cortex of urethane anesthetized rats. The calculated CSD maps of spontaneous up-states showed a broad current sink located in middle cortical layers, surrounded by a superficial and a deep layer current source. Only the first 50-ms-long period after up-state onset was examined in this study, but the obtained laminar profile showed high similarities with our results found in the somatosensory cortex. This suggests that the generator mechanisms underlying up-states may be very similar, at least in different sensory cortical areas. 
A recent study, supporting the similarity of SWA generation between different species, estimated the CSD distribution of ketamine/xylazine-induced slow waves in the neocortex of mice (Kuki et al., 2015). The pattern of transmembrane currents found in mice is nearly identical to our findings, except that the deep source they found was located in the CA1 region of the hippocampus. However, a precise anatomical examination was not the aim of the study of Kuki and colleagues (2015), therefore, based on our data, at least one part of this deep source could be located in layer VI.

Other studies support our findings only partially. A strong sink was found in layer V of the rat somatosensory cortex during delta waves, a brain rhythm with a frequency band (0.5-4 Hz) partially overlapping with the band of slow waves (Sirota et al., 2003). K-complexes occurring in the cortex during stage 2 of non-rapid eye movement sleep are thought to represent an isolated down-state (Cash et al., 2009). The CSD analysis of K-complexes recorded in the cortex of cats revealed a massive sink in cortical layers II and III surrounded by two sources, one located in superficial (layer I) and the other in the deeper layers (layer IV-V) (Amzica \& Steriade, 1998). As others (Toth et al., 2008; Chauvette et al., 2010), we have found that the pattern of transmembrane currents during down-states is the opposite of that observed during up-states, that is, strong superficial current sinks were present with current sources located in deep cortical layers.

Our results and the pieces of evidence mentioned above may suggest that the CSD depth distribution of cortical slow waves has very similar properties across different species (rat vs. cat vs. mouse), across different brain areas (somatosensory vs. auditory cortex) and across disparate anesthetic conditions (ketamine/xylazine vs. urethane vs. natural sleep). However, note that beside these similarities significant differences were also detected (e.g. deep cortical sink found under urethane anesthesia). 
$\underline{\text { Comparison of slow wave activity between rats and humans }}$

We compared our findings to the characteristics of SWA recorded during natural sleep in the neocortex of patients with drug-resistant focal epilepsy (Csercsa et al., 2010). In the human study, the LFP gradient was recorded instead of the LFP to minimize electro-magnetic artefacts (Ulbert et al., 2001; Csercsa et al., 2010). Therefore, we estimated the GRD from the LFP to facilitate a direct comparison between the recorded human and rat data. Some major differences between the results of the two studies can be observed. Comparing the up-state locked GRD averages, high amplitude positive waves were detected in both species in the supragranular layers with a layer II peak. However, this positivity had a greater spatial extent in rats reaching down to layer IV, while in humans it was restricted only to layer III. Based on the GRD depth profiles, only minor activity was detected in the infragranular layers in humans, contrary to ketamine/xylazine-induced slow-waves in rats, where negative amplitudes dominated the up-state onset in the deep layers, changing to positive values after the peak of the up-state. A sink-source pair was found in both species in the supragranular layers, where the sink reached into deeper layers accompanied with a deep source during the initiation phase of up-states in rats. In humans, only minimal infragranular currents were detected. The depth profile of the MUA was also different: rats had the strongest MUA in layer V during up-states, while in humans a layer III maximum was found beside a smaller layer V peak.

The differences in the results between rats and humans could originate from multiple sources, e.g. anesthesia in rats vs. natural sleep in humans, the recording methodology, cytoarchitectonics of the cortex (somatosensory cortex in rats vs. frontal and parietal areas in humans) or physiological differences between the species. The spectral peak of the human SWA during natural slow wave sleep was found around $1 \mathrm{~Hz}$, while ketamine/xylazine narcosis in rats induces slow waves with a higher peak frequency $(\sim 1.5 \mathrm{~Hz})$. However, the difference in frequency cannot account for the discrepancies found between rats and humans, since laminar 
analysis was carried out on human slow wave cycles with different durations, but no significant changes were observed between the groups (Csercsa et al., 2010). We also analyzed up-states with different durations in this study: qualitative properties of depth profile maps were similar between brief, average duration and long up-states.

The anesthetic conditions could also account for the differences between the two studies. Ketamine and xylazine act on several receptors in the brain (Drew et al., 1979; Hedler et al., 1981; Harrison \& Simmonds, 1985; MacDonald et al., 1991), which may alter the physiological expression of slow waves. Furthermore, several differences were found between SWA during natural sleep and anesthesia in the same animals with the same recording and analyzing methods (Chauvette et al., 2011).

Phylogenetic differences may explain some of the differences found in the CSD and MUA depth profiles during up-states. The human neocortex is considerably thicker compared to the rat neocortex $(2.5 \mathrm{~mm}$ vs $1.8 \mathrm{~mm})$ and the density of neurons is higher in the thinner cortex of rats (Defelipe, 2011). Furthermore, while infragranular layers in the rodent primary somatosensory cortex account for more than the half of the cortical thickness, supragranular layers in the primate and human S1 are more developed compared to infragranular layers (Defelipe, 2011). These facts may contribute to the dominance of supragranular layers in the generation of SWA found in the human study.

Of course, we can not exclude the possibility that some of the observed differences may be the result of the patients' pathology. Even though care was taken to avoid analyzing data in the temporal proximity of epileptic seizures, furthermore, the investigated properties of SWA were comparable to previous findings in healthy subjects (Massimini et al., 2004), epileptic networks may have contributed to the expression of the recorded slow waves (Csercsa et al., 2010). However, the lack of any MRI abnormalities and intact laminarization of the excised 
tissue also suggest that recordings were performed in structurally intact regions (Csercsa et al., 2010).

$\underline{\text { The prominent role of cortical layer } \mathrm{V} \text { during up-states in animals }}$

The firing rate of neocortical neurons is the highest in layer V, while layer II/III shows ‘sparse' firing (Sanchez-Vives \& McCormick, 2000; Manns et al., 2004; de Kock et al., 2007; de Kock \& Sakmann, 2009; Sakata \& Harris, 2009; Barth \& Poulet, 2012; Petersen \& Crochet, 2013). Similar to these studies, we have found the strongest MUA during up-states in layer V of the somatosensory cortex, while a weaker firing activity was present in the supragranular layers.

Our in vivo measurements suggest that up-state related MUA starts most frequently in layer $\mathrm{V}$, similar to extracellular recordings from ferret neocortical slices generating slow waves (Sanchez-Vives \& McCormick, 2000). In this in vitro model of SWA, cell firing was most frequently initiated in layer $\mathrm{V}$ which then spread to deeper and superficial layers. Another study found that in naturally sleeping cats the earliest firing is located in layer $\mathrm{V}$ and this layer also showed the highest firing probability (Chauvette et al., 2010). On laminar MUA recordings from the auditory cortex of urethane anesthetized rats, the up-state onset was first detected in deeper layers which then spread towards upper layers (Sakata \& Harris, 2009). Furthermore, based on various information theoretical indicators, Amigo and colleagues (2015) found that infragranular layers lead the information flow during slow waves towards supragranular layers. All the above information point toward the crucial role of firing of layer $\mathrm{V}$ neurons in initiating slow wave activity in animals, independent of species, experimental methods and brain region. Large pyramidal cells in layer $\mathrm{V}$ are among the largest neurons in the brain, with a dendritic tree usually reaching the top of the cortex. These neurons receive a high number of synaptic inputs, have large projection fields and a powerful output connectivity to all other 
layers in a cortical column, and are therefore well suited to change the current state of the network (Chauvette et al., 2010). A recent study found electrophysiologically distinct subsets of layer $\mathrm{V}$ intrinsically bursting and regular spiking pyramidal neurons which might have a significant role in the initiation and driving of the up-states (Lorincz et al., 2015). Studies using optogenetic methods also support the importance of layer $\mathrm{V}$ pyramidal cells in the generation of cortical up-states (Beltramo et al., 2013; Kuki et al., 2013; Stroh et al., 2013). Furthermore, layer V neurons are intrinsically more depolarized than layer II/III neurons (Lefort et al., 2009), which implies that layer $\mathrm{V}$ neurons have a higher probability to fire first during up-state onsets. Another study modeling the rodent neocortical column and simulating the LFP in the cortex states that layer V pyramidal cells could also significantly contribute to the LFP in other layers (Reimann et al., 2013). Because of the dominating MUA in layer V, we can assume that the CSD profile observed by us is strongly influenced by the activity of large pyramidal cells located in layer $\mathrm{V}$.

The influence of the thalamus during slow wave activity

Repetitive sensory stimulation can entrain the cortical slow wave activity and can evoke either up- or down-states, depending on the current state of the cortex and the parameters of stimulation (Shu et al., 2003; Hasenstaub et al., 2007). Based on the CSD or MUA depth profile of evoked up-states in our experiments, the onset of evoked up-states was mainly detected in the thalamorecipient layer IV, while the onset of spontaneously occurring up-states was found most frequently in layer V. Furthermore, in several experiments, the ratio of spontaneous upstates with layer IV onset (putative thalamus-driven up-states) was higher than that of up-states with layer $\mathrm{V}$ onset. These results suggest that certain fraction of the up-states could be initiated by the thalamus in cortical areas having a strong reciprocal connection with the thalamus. This may indicate that specific areas exist in the somatosensory cortex, where subcortical (thalamic) 
activity is able to influence the states of the cortical activity and trigger new slow waves. In humans, cortical hot spots were found, where up-states tend to begin with a higher possibility (Murphy et al., 2009). These hot spots (insula and cingulate gyrus) have strong connections with the thalamus (Baleydier \& Mauguiere, 1980; Augustine, 1985; 1996), therefore, a significant number of cortical slow waves might have been initiated by the thalamus.

Another possible explanation for the presence of putative thalamic-originating up-states could be that the investigated regions of the somatosensory cortex receive a continuous respiration-induced skin stimulation, which in turn evokes cortical up-states. These evoked upstates are then classified falsely as spontaneously occurring up-states. Also, the applied mechanical skin stimulation may have an aftereffect, that is, thalamocortical pathways might be activated for longer time scales due to stimulation, which, in turn, can result in a more frequent occurrence of cortical up-states with layer IV onset.

Our findings are in line with the growing scientific evidence indicating that the cortex is not the sole generator of the SWA, but the thalamus can also have an important role in the generation and shaping of slow waves (Crunelli \& Hughes, 2010; Gemignani et al., 2012; David et al., 2013; Crunelli et al., 2015; Lemieux et al., 2014). 


\section{Acknowledgements}

The authors wish to thank Katalin Lengyel for her help during histological procedures and Péter Kottra for his excellent technical assistance. We are grateful to Katharina T. Hofer for useful comments on the manuscript. This work was supported by the EU FP7 grant 600925, NeuroSeeker and by the Hungarian Brain Research Program grant KTIA-13-NAP-A-IV/1-4,6. The authors declare no conflict of interest. 


\begin{abstract}
Abbreviations
CSD, current-source density; DiI, 1,1'-Dioctadecyl 3,3,3',3'-Tetramethylindocarbocyanine Perchlorate; FFT, fast Fourier transform; GRD, local field potential gradient; LFP, local field potential; MUA, multiple-unit activity; S1HL, hindlimb region of the primary somatosensory cortex; S1Tr, trunk region of the primary somatosensory cortex; SPA, summated population activity; SWA, slow wave activity;
\end{abstract}




\section{References}

Achermann, P. \& Borbely, A.A. (1997) Low-frequency $(<1 \mathrm{~Hz})$ oscillations in the human sleep electroencephalogram. Neuroscience, 81, 213-222.

Amigo, J.M., Monetti, R., Tort-Colet, N. \& Sanchez-Vives, M.V. (2015) Infragranular layers lead information flow during slow oscillations according to information directionality indicators. J. Comput. Neurosci., 39, 53-62.

Amzica, F. \& Steriade, M. (1998) Cellular substrates and laminar profile of sleep K-complex. Neuroscience, 82, 671-686.

Augustine, J.R. (1985) The insular lobe in primates including humans. Neurol. Res., 7, 2-10.

Augustine, J.R. (1996) Circuitry and functional aspects of the insular lobe in primates including humans. Brain Res. Rev., 22, 229-244.

Baleydier, C. \& Mauguiere, F. (1980) The duality of the cingulate gyrus in monkey. Neuroanatomical study and functional hypothesis. Brain, 103, 525-554.

Barth, A.L. \& Poulet, J.F. (2012) Experimental evidence for sparse firing in the neocortex. Trends Neurosci., 35, 345-355.

Beltramo, R., D'Urso, G., Dal Maschio, M., Farisello, P., Bovetti, S., Clovis, Y., Lassi, G., Tucci, V., De Pietri Tonelli, D. \& Fellin, T. (2013) Layer-specific excitatory circuits differentially control recurrent network dynamics in the neocortex. Nat. Neurosci., 16, 227-234.

Born, J., Rasch, B. \& Gais, S. (2006) Sleep to remember. Neuroscientist, 12, 410-424.

Cash, S.S., Halgren, E., Dehghani, N., Rossetti, A.O., Thesen, T., Wang, C., Devinsky, O., Kuzniecky, R., Doyle, W., Madsen, J.R., Bromfield, E., Eross, L., Halasz, P., Karmos, G., Csercsa, R., Wittner, L. \& Ulbert, I. (2009) The human K-complex represents an isolated cortical down-state. Science, 324, 1084-1087.

Chagnac-Amitai, Y., Luhmann, H.J. \& Prince, D.A. (1990) Burst generating and regular spiking layer 5 pyramidal neurons of rat neocortex have different morphological features. J. Comp. Neurol., 296, 598-613. 
Chauvette, S., Crochet, S., Volgushev, M. \& Timofeev, I. (2011) Properties of slow oscillation during slow-wave sleep and anesthesia in cats. J. Neurosci., 31, 1499815008 .

Chauvette, S., Volgushev, M. \& Timofeev, I. (2010) Origin of active states in local neocortical networks during slow sleep oscillation. Cereb. Cortex, 20, 2660-2674.

Crunelli, V., David, F., Lorincz, M.L. \& Hughes, S.W. (2015) The thalamocortical network as a single slow wave-generating unit. Curr. Opin. Neurobiol., 31, 72-80.

Crunelli, V. \& Hughes, S.W. (2010) The slow $(<1 \mathrm{~Hz})$ rhythm of non-REM sleep: a dialogue between three cardinal oscillators. Nat. Neurosci., 13, 9-17.

Csercsa, R., Dombovari, B., Fabo, D., Wittner, L., Eross, L., Entz, L., Solyom, A., Rasonyi, G., Szucs, A., Kelemen, A., Jakus, R., Juhos, V., Grand, L., Magony, A., Halasz, P., Freund, T.F., Magloczky, Z., Cash, S.S., Papp, L., Karmos, G., Halgren, E. \& Ulbert, I. (2010) Laminar analysis of slow wave activity in humans. Brain, 133, 2814-2829.

David, F., Schmiedt, J.T., Taylor, H.L., Orban, G., Di Giovanni, G., Uebele, V.N., Renger, J.J., Lambert, R.C., Leresche, N. \& Crunelli, V. (2013) Essential Thalamic Contribution to Slow Waves of Natural Sleep. J. Neurosci., 33, 19599-19610.

Defelipe, J. (2011) The evolution of the brain, the human nature of cortical circuits, and intellectual creativity. Front. Neuroanat., 5, 29.

de Kock, C.P., Bruno, R.M., Spors, H. \& Sakmann, B. (2007) Layer- and cell-type-specific suprathreshold stimulus representation in rat primary somatosensory cortex. $J$. Physiol., 581, 139-154.

de Kock, C.P. \& Sakmann, B. (2009) Spiking in primary somatosensory cortex during natural whisking in awake head-restrained rats is cell-type specific. Proc. Natl. Acad. Sci. U. S. A., 106, 16446-16450.

Delorme, A. \& Makeig, S. (2004) EEGLAB: an open source toolbox for analysis of singletrial EEG dynamics including independent component analysis. J. Neurosci. Methods, 134, 9-21.

Drew, G.M., Gower, A.J. \& Marriott, A.S. (1979) Alpha 2-adrenoceptors mediate clonidineinduced sedation in the rat. Br. J. Pharmacol., 67, 133-141. 
Einevoll, G.T., Pettersen, K.H., Devor, A., Ulbert, I., Halgren, E. \& Dale, A.M. (2007) Laminar population analysis: estimating firing rates and evoked synaptic activity from multielectrode recordings in rat barrel cortex. J. Neurophysiol., 97, 2174-2190.

Freeman, J.A. \& Nicholson, C. (1975) Experimental optimization of current source-density technique for anuran cerebellum. J. Neurophysiol., 38, 369-382.

Gemignani, A., Laurino, M., Provini, F., Piarulli, A., Barletta, G., d'Ascanio, P., Bedini, R., Lodi, R., Manners, D.N., Allegrini, P., Menicucci, D. \& Cortelli, P. (2012) Thalamic contribution to Sleep Slow Oscillation features in humans: a single case cross sectional EEG study in Fatal Familial Insomnia. Sleep Med., 13, 946-952.

Grand, L., Pongrácz, A., Vázsonyi, E., Márton, G., Gubán, D., Fiáth, R., Kerekes, B.P., Karmos, G., Ulbert, I. \& Battistig, G. (2011) A novel multisite silicon probe for high quality laminar neural recordings. Sensors and Actuators, A: Physical, 166, 14-21.

Gulyas, A.I., Megias, M., Emri, Z. \& Freund, T.F. (1999) Total number and ratio of excitatory and inhibitory synapses converging onto single interneurons of different types in the CA1 area of the rat hippocampus. J. Neurosci., 19, 10082-10097.

Harrison, N.L. \& Simmonds, M.A. (1985) Quantitative studies on some antagonists of Nmethyl D-aspartate in slices of rat cerebral cortex. Br. J. Pharmacol., 84, 381-391.

Hasenstaub, A., Sachdev, R.N. \& McCormick, D.A. (2007) State changes rapidly modulate cortical neuronal responsiveness. J. Neurosci., 27, 9607-9622.

Hedler, L., Stamm, G., Weitzell, R. \& Starke, K. (1981) Functional characterization of central alpha-adrenoceptors by yohimbine diastereomers. Eur. J. Pharmacol., 70, 43-52.

Kasper, E.M., Larkman, A.U., Lubke, J. \& Blakemore, C. (1994) Pyramidal neurons in layer 5 of the rat visual cortex. I. Correlation among cell morphology, intrinsic electrophysiological properties, and axon targets. J. Comp. Neurol., 339, 459-474.

Kuki, T., Ohshiro, T., Ito, S., Ji, Z.G., Fukazawa, Y., Matsuzaka, Y., Yawo, H. \& Mushiake, H. (2013) Frequency-dependent entrainment of neocortical slow oscillation to repeated optogenetic stimulation in the anesthetized rat. Neurosci. Res., 75, 35-45.

Kuki, T., Fujihara, K., Miwa, H., Tamamaki, N., Yanagawa, Y. \& Mushiake, H. (2015) Contribution of parvalbumin and somatostatin-expressing GABAergic neurons to slow oscillations and the balance in beta-gamma oscillations across cortical layers. Front. Neural Circuits, 9, 6. 
Lefort, S., Tomm, C., Floyd Sarria, J.C. \& Petersen, C.C. (2009) The excitatory neuronal network of the $\mathrm{C} 2$ barrel column in mouse primary somatosensory cortex. Neuron, $\mathbf{6 1}$, 301-316.

Lemieux, M., Chauvette, S. \& Timofeev, I. (2015) Neocortical inhibitory activities and longrange afferents contribute to the synchronous onset of silent states of the neocortical slow oscillation. J. Neurophysiol., 113, 768-779.

Lemieux, M., Chen, J.Y., Lonjers, P., Bazhenov, M. \& Timofeev, I. (2014) The Impact of Cortical Deafferentation on the Neocortical Slow Oscillation. J. Neurosci., 34, 56895703.

Lorincz, M.L., Gunner, D., Bao, Y., Connelly, W.M., Isaac, J.T., Hughes, S.W. \& Crunelli, V. (2015) A Distinct Class of Slow (approximately 0.2-2 Hz) Intrinsically Bursting Layer 5 Pyramidal Neurons Determines UP/DOWN State Dynamics in the Neocortex. J. Neurosci., 35, 5442-5458.

Luczak, A. \& Bartho, P. (2012) Consistent sequential activity across diverse forms of UP states under ketamine anesthesia. Eur. J. Neurosci., 36, 2830-2838.

MacDonald, J.F., Bartlett, M.C., Mody, I., Pahapill, P., Reynolds, J.N., Salter, M.W., Schneiderman, J.H. \& Pennefather, P.S. (1991) Actions of ketamine, phencyclidine and MK-801 on NMDA receptor currents in cultured mouse hippocampal neurones. $J$. Physiol., 432, 483-508.

Manns, I.D., Sakmann, B. \& Brecht, M. (2004) Sub- and suprathreshold receptive field properties of pyramidal neurones in layers $5 \mathrm{~A}$ and $5 \mathrm{~B}$ of rat somatosensory barrel cortex. J. Physiol., 556, 601-622.

Massimini, M., Huber, R., Ferrarelli, F., Hill, S. \& Tononi, G. (2004) The sleep slow oscillation as a traveling wave. J. Neurosci., 24, 6862-6870.

Massimini, M., Ferrarelli, F., Esser, S.K., Riedner, B.A., Huber, R., Murphy, M., Peterson, M.J. \& Tononi, G. (2007) Triggering sleep slow waves by transcranial magnetic stimulation. Proc. Natl. Acad. Sci. U. S. A., 104, 8496-8501.

Mitzdorf, U. (1985) Current source-density method and application in cat cerebral cortex: investigation of evoked potentials and EEG phenomena. Physiol. Rev., 65, 37-100.

Mukovski, M., Chauvette, S., Timofeev, I. \& Volgushev, M. (2007) Detection of active and silent states in neocortical neurons from the field potential signal during slow-wave sleep. Cereb. Cortex, 17, 400-414. 
Murphy, M., Riedner, B.A., Huber, R., Massimini, M., Ferrarelli, F. \& Tononi, G. (2009) Source modeling sleep slow waves. Proc. Natl. Acad. Sci. U. S. A., 106, 1608-1613.

Nicholson, C. \& Freeman, J.A. (1975) Theory of current source-density analysis and determination of conductivity tensor for anuran cerebellum. J. Neurophysiol., 38, 356368 .

Paxinos, G. \& Watson, C. (2007) The Rat Brain in Stereotaxic Coordinates, 6th Edn. Academic Press, San Diego.

Petersen, C.C. \& Crochet, S. (2013) Synaptic computation and sensory processing in neocortical layer 2/3. Neuron, 78, 28-48.

Pyapali, G.K., Sik, A., Penttonen, M., Buzsaki, G. \& Turner, D.A. (1998) Dendritic properties of hippocampal CA1 pyramidal neurons in the rat: intracellular staining in vivo and in vitro. J. Comp. Neurol., 391, 335-352.

Rappelsberger, P., Pockberger, H. \& Petsche, H. (1981) Current source density analysis: methods and application to simultaneously recorded field potentials of the rabbit's visual cortex. Pflugers Arch., 389, 159-170.

Rasch, B. \& Born, J. (2013) About sleep's role in memory. Physiol. Rev., 93, 681-766.

Reimann, Michael W., Anastassiou, Costas A., Perin, R., Hill, S.L., Markram, H. \& Koch, C. (2013) A Biophysically Detailed Model of Neocortical Local Field Potentials Predicts the Critical Role of Membrane Currents. Neuron, 79, 375-390.

Sakata, S. \& Harris, K.D. (2009) Laminar structure of spontaneous and sensory-evoked population activity in auditory cortex. Neuron, 64, 404-418.

Saleem, A.B., Chadderton, P., Apergis-Schoute, J., Harris, K.D. \& Schultz, S.R. (2010) Methods for predicting cortical UP and DOWN states from the phase of deep layer local field potentials. J. Comput. Neurosci., 29, 49-62.

Sanchez-Vives, M.V., Mattia, M., Compte, A., Perez-Zabalza, M., Winograd, M., Descalzo, V.F. \& Reig, R. (2010) Inhibitory modulation of cortical up states. J. Neurophysiol., 104, 1314-1324.

Sanchez-Vives, M.V. \& McCormick, D.A. (2000) Cellular and network mechanisms of rhythmic recurrent activity in neocortex. Nat. Neurosci., 3, 1027-1034. 
Schubert, D., Kotter, R., Luhmann, H.J. \& Staiger, J.F. (2006) Morphology, electrophysiology and functional input connectivity of pyramidal neurons characterizes a genuine layer va in the primary somatosensory cortex. Cereb. Cortex, 16, 223-236.

Sharma, A.V., Wolansky, T. \& Dickson, C.T. (2010) A comparison of sleeplike slow oscillations in the hippocampus under ketamine and urethane anesthesia. $J$. Neurophysiol., 104, 932-939.

Sheroziya, M. \& Timofeev, I. (2014) Global intracellular slow-wave dynamics of the thalamocortical system. J. Neurosci., 34, 8875-8893.

Shu, Y., Hasenstaub, A. \& McCormick, D.A. (2003) Turning on and off recurrent balanced cortical activity. Nature, 423, 288-293.

Sirota, A., Csicsvari, J., Buhl, D. \& Buzsaki, G. (2003) Communication between neocortex and hippocampus during sleep in rodents. Proc. Natl. Acad. Sci. U. S. A., 100, 20652069.

Steriade, M. \& Amzica, F. (1996) Intracortical and corticothalamic coherency of fast spontaneous oscillations. Proc. Natl. Acad. Sci. U. S. A., 93, 2533-2538.

Steriade, M., Contreras, D., Curro Dossi, R. \& Nunez, A. (1993a) The slow (<1 Hz) oscillation in reticular thalamic and thalamocortical neurons: scenario of sleep rhythm generation in interacting thalamic and neocortical networks. J. Neurosci., 13, 32843299.

Steriade, M., Nunez, A. \& Amzica, F. (1993b) Intracellular analysis of relations between the slow $(<1 \mathrm{~Hz})$ neocortical oscillation and other sleep rhythms of the electroencephalogram. J. Neurosci., 13, 3266-3283.

Steriade, M., Nunez, A. \& Amzica, F. (1993c) A novel slow $(<1 \mathrm{~Hz})$ oscillation of neocortical neurons in vivo: depolarizing and hyperpolarizing components. $J$. Neurosci., 13, 3252-3265.

Steriade, M., Timofeev, I. \& Grenier, F. (2001) Natural waking and sleep states: A view from inside neocortical neurons. J. Neurophysiol., 85, 1969-1985.

Stroh, A., Adelsberger, H., Groh, A., Ruhlmann, C., Fischer, S., Schierloh, A., Deisseroth, K. \& Konnerth, A. (2013) Making waves: initiation and propagation of corticothalamic $\mathrm{Ca} 2+$ waves in vivo. Neuron, 77, 1136-1150. 
Tononi, G. \& Cirelli, C. (2006) Sleep function and synaptic homeostasis. Sleep Med. Rev., 10, 49-62.

Tononi, G. \& Cirelli, C. (2014) Sleep and the price of plasticity: from synaptic and cellular homeostasis to memory consolidation and integration. Neuron, 81, 12-34.

Toth, A., Gyengesi, E., Zaborszky, L. \& Detari, L. (2008) Interaction of slow cortical rhythm with somatosensory information processing in urethane-anesthetized rats. Brain Res., 1226, 99-110.

Townsend, G., Peloquin, P., Kloosterman, F., Hetke, J.F. \& Leung, L.S. (2002) Recording and marking with silicon multichannel electrodes. Brain Res. Brain Res. Protoc., 9 , 122-129.

Tukker, J.J., Lasztoczi, B., Katona, L., Roberts, J.D., Pissadaki, E.K., Dalezios, Y., Marton, L., Zhang, L., Klausberger, T. \& Somogyi, P. (2013) Distinct dendritic arborization and in vivo firing patterns of parvalbumin-expressing basket cells in the hippocampal area CA3. J. Neurosci., 33, 6809-6825.

Turner, D.A., Li, X.G., Pyapali, G.K., Ylinen, A. \& Buzsaki, G. (1995) Morphometric and Electrical-Properties of Reconstructed Hippocampal Ca3 Neurons Recorded in-Vivo. J. Comp. Neurol., 356, 580-594.

Ulbert, I., Halgren, E., Heit, G. \& Karmos, G. (2001) Multiple microelectrode-recording system for human intracortical applications. J. Neurosci. Methods, 106, 69-79.

Volgushev, M., Chauvette, S., Mukovski, M. \& Timofeev, I. (2006) Precise long-range synchronization of activity and silence in neocortical neurons during slow-wave oscillations. The Journal of neuroscience : the official journal of the Society for Neuroscience, 26, 5665-5672.

Vyazovskiy, V.V., Faraguna, U., Cirelli, C. \& Tononi, G. (2009) Triggering slow waves during NREM sleep in the rat by intracortical electrical stimulation: effects of sleep/wake history and background activity. J. Neurophysiol., 101, 1921-1931.

Wellman, C.L., Logue, S.F. \& Sengelaub, D.R. (1995) Maze learning and morphology of frontal cortex in adult and aged basal forebrain-lesioned rats. Behav. Neurosci., 109, 837-850.

Wester, J.C. \& Contreras, D. (2012) Columnar interactions determine horizontal propagation of recurrent network activity in neocortex. J. Neurosci., 32, 5454-5471. 
Wilson, C.J. \& Kawaguchi, Y. (1996) The origins of two-state spontaneous membrane potential fluctuations of neostriatal spiny neurons. J. Neurosci., 16, 2397-2410. 